\title{
The Exactness Theorem for Floer Homology
}

\author{
By
}

\author{
Hong-Yu WANG*
}

\begin{abstract}
In this article. we study a long exact sequence relating the instanton homology of two homology 3 -spheres which are obtained from each other by \pm 1 -surgery. We prove that the long exact sequence, via a short exact sequence of chain complex, is the same as the sequence defined by the exact triangle of cobordisms introduced by Floer.
\end{abstract}

One development which has attracted a great deal of attention has been Floer's work on "instanton homology" of 3-manifolds [10], [14], [15], [19], [20]. The basic idea is to find Floer homology groups $H F_{*}(Y)$ associated to an oriented 3 -manifold $Y$ by studying instantons on the tube $Y \times \mathbb{R}$. The theory applies in the first instance to homology 3-sphere $Y$. A fundamental question in Floer theory is the calculation of the Floer homology groups. A great step forward here was made by Floer who found an "exact triangle" of homomorphisms

$$
\cdots H F_{*}\left(Y^{\prime}\right) \rightarrow H F_{*}(Y) \rightarrow H F_{*}\left(Y^{\prime \prime}\right) \rightarrow H F_{*-1}\left(Y^{\prime}\right) \rightarrow \cdots
$$

connecting the Floer homology groups of the 3-manifold $Y$ with those of the 3-manifolds $Y^{\prime}, Y^{\prime \prime}$ obtained from $Y$ by Dehn surgery on a knot [6], [16], [17], [18].

This paper is based on the works of Floer, Braam and Donaldson. We consider a long exact sequence relating the instanton homology of two homology 3 -spheres which are obtained from each other by \pm 1 -surgery. The third term is a $\mathbb{Z}_{4}$-graded homology of the homology $S^{1} \times S^{2}$ which is associated to a knot in the homology 3 -sphere via 0 -surgery. We prove that the long exact sequence obtained via a short exact sequence of chain complex, is the same as the sequence defined by the exact triangle of cobordisms introduced by Floer in [16], [17].

Suppose that, as above, $Y$ is an oriented homology 3 -sphere and $\mathscr{K} \subset Y$ is a

Communicated by K. Saito, October 16, 1995.

1991 Mathematics Subject Classification (s) : 57N10, 55N35

*Department of Mathematics, National University of Singapore, Singapore 119260 
knot, that is, a smoothly embedded circle in $Y$. We introduce two other 3-manifolds:

(1) The homology 3 -sphere $Y^{\prime}$ obtained from $Y$ by +1 -surgery on $\mathscr{K}$,

(2) The homology $S^{1} \times S^{2} Y^{\prime \prime}$ obtained from $Y$ by 0 -surgery on $\mathscr{K}$.

There are standard, oriented, surgery cobordisms $W_{1}$ from $Y^{\prime}$ to $Y$ and $W_{2}$ from $Y$ to $Y^{\prime \prime}$. Also there is a cobordism $W_{3}$ from $Y^{\prime \prime}$ to $Y^{\prime}$ via the composition of $W_{1}$ and $W_{2}$.

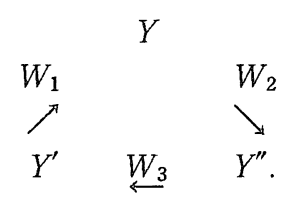

We now consider the Floer homology of the three 3-manifolds $Y^{\prime}, Y, Y^{\prime \prime}$. The cobordisms $W_{1}, W_{2}$ and $W_{3}$ induce the maps $\Psi_{W_{1}}, \Psi_{W_{2}}$, and $\Psi_{W_{3}}$ on the Floer homology groups on $Y^{\prime}, Y$ and $Y^{\prime \prime}$. We shall abbreviate $\Psi_{W_{1}}, \Psi_{W_{2}}$, and $\Psi_{W_{3}}$ to $a_{*}, b_{*}$, and $c_{*}$ respectively. Combining the works of Floer, Braam and Donaldson, we have the following theorem:

Theorem. Let $a, b$ and $c$ be the chain maps between the chain complexes on $Y^{\prime}, Y$ and $Y^{\prime \prime}$ induced by the cobordisms $W_{1}, W_{2}$ and $W_{3}$ respectively, as follows:

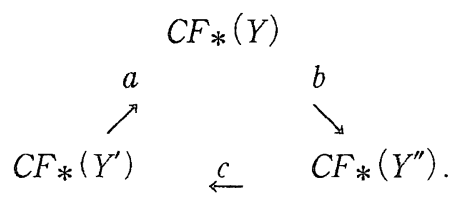

We have the following properties:

(1) The maps $a_{*}, b_{*}$ have degree 0 , with respect to the grading; but the map $c_{*}$ has degree -1 , with respect to the grading.

(2) The maps $a_{*}, b_{*}$ and $c *$ induce a long exact sequence, or exact triangle.

$$
\cdots H F_{\imath+1}\left(Y^{\prime \prime}\right) \stackrel{c_{*}}{\rightarrow} H F_{\imath}\left(Y^{\prime}\right) \stackrel{a_{*}}{\rightarrow} H F_{\imath}(Y) \stackrel{b_{*}}{\rightarrow} H F_{\imath}\left(Y^{\prime \prime}\right) \stackrel{c_{*}}{\rightarrow} H F_{\imath-1}\left(Y^{\prime}\right) \cdots .
$$

(3) By using deformations, one may find maps

$$
\widetilde{a}: C F *\left(Y^{\prime}\right) \rightarrow \widetilde{C F}_{*}(Y), \quad \tilde{b}: \widetilde{C F}_{*}(Y) \rightarrow C F *\left(Y^{\prime \prime}\right)
$$

which are chain homotopic to $a$ and $b$ respectively. Simultaneonsly, $\overline{C F}_{*}(\mathrm{Y})$ is isomorphic to the direct sum of $C F_{*}\left(\mathrm{Y}^{\prime}\right)$ and $C F_{*}\left(\mathrm{Y}^{\prime \prime}\right)$,

$$
\overline{C F}_{*}(\mathrm{Y}) \cong C F *\left(Y^{\prime}\right) \oplus C F_{*}\left(Y^{\prime \prime}\right) .
$$

(4) The composite $\tilde{b} \widetilde{a}$ is chain homotopic to zero by a homotopy $H: C F_{*}\left(Y^{\prime}\right) \rightarrow$ 
$C F_{*}\left(Y^{\prime \prime}\right)$ (i.e. $\left.\partial H+H \partial=\tilde{b} \tilde{a}\right)$, where $\partial$ is a boundary operator of the chain complexes. Then there is a long exact homology sequence,

$$
\cdots \rightarrow H F_{i}\left(Y^{\prime}\right) \stackrel{a_{*}}{\rightarrow} H F_{\imath}(Y) \stackrel{b_{*}}{\rightarrow} H F_{\imath}\left(Y^{\prime \prime}\right) \stackrel{\Delta_{+}}{\rightarrow} H F_{i-1}\left(Y^{\prime}\right) \rightarrow \cdots,
$$

where $\Delta_{*}$ is induced by the short exact sequence,

$$
0 \rightarrow C F *\left(Y^{\prime}\right) \stackrel{\tilde{a}_{1}}{\rightarrow} \widetilde{C F} *(Y) \stackrel{\tilde{b}}{\rightarrow} C F *\left(Y^{\prime \prime}\right) \rightarrow 0,
$$

and the boundary operator $\partial$ of the chain complexes, and where $\widetilde{a}_{1}$ is chain homotopic to $\tilde{a}$.

(5) The long exact sequence in (4) above, via the short exact sequence of the chain complexes, is actually the same as the sequence in (2) defined by the triangle of the cobordisms, that is, $\triangle_{*}=c_{*}$.

It is worth remarking here: (1) The definition of $H F_{*}$ [15] suggests that one should consider it as a homology theory, and we shall in fact refer to it as the instanton homology. It is the purpose of the present paper to expose further properties of $H F *$ to justify this terminology. (2) Parts 1 to 4 of the above Theorem are due to Floer [17], Braam and Donaldson [6]. Part 5, $\triangle_{*}$ $=c *$ is the main result of this paper. Here $\widetilde{H F}_{*}(Y) \cong H F_{*}(Y)$ where $\widetilde{H F}_{*}(Y)$ is the homology of the complex $\widetilde{C F}_{*}(Y)$ and $\widetilde{a}_{1 *}=a_{*}, \tilde{b}_{*}=b *$ since $\tilde{a}$ and $\tilde{b}$ are chain homotopic to $a$ and $b$ respectively. (3) Floer in [16], [18] developed a more general framework for the calculation scheme of Floer homology, the exact triangle was discussed in the general setting, we shall consider this subject in a subsequent paper. (4) This paper should be considered as a sequel to [6], [16] and [17], where most of the notation and the terminology were introduced. The reader may find the expositions in [5], [10] and [15] useful as an introduction to the instanton homology.

This paper is organized as follows. In $\S 1$ and 2 we give an introduction to the relevant geometry and topology of the space of connections on a 3 -manifold. In $\S 3$, we describe the basic features of the Dehn surgery, the grading of the chain complex and some useful computations. $\S 4$ discusses the deformations of instanton equations, geometric triangle and Morse-Smale type flow. In $\S 5$, we investigate the Floer exact triangle, compare the Floer exact triangle with the long exact sequence described by Braam and Donaldson, and prove the main theorem. Finally, the appendix is a review of the index formulae.

Before turning to a detailed description, some acknowledgments are due: The approach in this article was suggested by S. K. Donaldson, P. Braam assisted us in understanding Floer's work and S. P. Tan, Y. L. Wong gave us useful comments and suggestions. Further acknowledgments to the 
Mathematical Institute of Oxford University and S. K. Donaldson; and Mathematics Department of Peking University and K. C. Chang for their generosity, kindness and hospitality, where much of this work was carried out.

\section{§1. Background}

We begin by reviewing some fundamental points about the Floer homology groups for 3-manifolds (in particular for homology 3-spheres). These fundamentals are now widely known: More details and background can be found in Floer's original paper [15], in the exposition of [5], the forthcoming book [10], and good survey of Atiyah [2]. Let $Y$ be an oriented homology 3-sphere. We work with the trivial $S U(2)$ bundle and $A S D$ connections

$$
F=-* F
$$

over the product manifold $Y \times \mathbb{R}$. To define Floer homology on $Y$, consider the trivial $S U(2)$ bundle $P \rightarrow Y . P$ has connections over $Y$, the flat $S U(2)$ connections over $Y$ may be regarded as the critical points of the Chern-Simons functional on the connection space $\mathscr{A}(P)$ :

$$
\begin{gathered}
\forall a \in \mathscr{A}(P), \\
\mathscr{C} \&(a)=\frac{1}{8 \pi^{2}} \int_{Y} \operatorname{tr}\left(a \wedge d a+\frac{2}{3} a \wedge a \wedge a\right) .
\end{gathered}
$$

Let $R(Y)$ denote the critical set of Chern-Simons with equivalence. It is well known that the homology yields an injective map

$$
R(P) \rightarrow \operatorname{Hom}\left(\pi_{1}(Y), S U(2)\right) / \operatorname{ad}(S U(2)) .
$$

The flat connections are therefore sometimes referred to as representations (of the fundamental group). Conversely, for each representation one can construct an $S U(2)$-bundle with a flat connection whose homology is prescribed by the representation [1].

It is $R(Y)$ which will become the set of simplexes in the instanton homology. To understand this, recall the following construction of the relative Morse theory (cf. [2], [5], [6], [15] and [32]).

For flat connections $\sigma, \rho \in R(Y)$, let $A$ be an $A S D$ connection over the tube $Y \times \mathscr{R}$ which is asymptotic to $\rho$ and $\sigma$ respectively at $-\infty$ and $+\infty$. Consider the operator $d_{A}^{+}+d_{A}^{*}$ coupled to the connection $A$ over the tube. The virtual dimension of the module space, $\mathcal{M}(\rho, \sigma)$, of such $A S D$ connections is equal to the relative index ind $(\rho, \sigma) \bmod 8$. For a generic choice of perturbation and metric on $Y$ the moduli space is a smooth manifold of this dimension. If $\sigma$ is not equal to $\rho$, then the translations of the tube act freely on the moduli space so we get a reduced space $\mathcal{M}_{0}(\rho, \sigma)=\mathcal{M}(\rho, \sigma) / \mathbb{R}$. 
To define the Floer homology groups $H F_{*}(Y)$ on $Y$, one forms the $\mathbb{Z}_{8}$-graded chain complex with groups

$$
C F_{\imath}(Y)=\bigoplus_{\rho \in R_{t}(Y)} \mathbb{Z}<\rho>
$$

generated by the critical points $R_{\imath}(Y)$ where $R_{\imath}$ is the subset of equivalence flat connection classes over $Y$ with grading $i$. Then the Floer differential

$$
\partial: C F_{\imath}(Y) \longrightarrow C F_{\imath-1}(Y)
$$

is defined by

$$
\partial<\rho>=\sum_{\sigma \in R_{t-1}(Y)} n(\rho, \sigma)<\sigma>
$$

where $n(\rho, \sigma)$ is the number of points in the 0 -dimensional space $\mathcal{M}_{0}(\rho, \sigma)$, counted with appropriate signs. The main result is that $\partial^{2}=0$ and the homology groups $H F_{*}(Y)$ of $\left(C F_{*}(Y), \partial\right)$ are independent of the choice of perturbation and metric.

These Floer homology groups allow us to extend the Donaldson's invariant for closed 4-manifolds to 4-manifolds with boundary. Consider an oriented 4-manifold $X$ with boundary a homology 3 -sphere $Y$. Under suitable conditions on $X$, there is a moduli space $\mathcal{M}(X ; \rho)$ of instantons, over the 4-manifold obtained by adjoining a semi-infinite tube $Y \times[0, \infty)$ to $X$, with limit $\rho \in R(Y)$, having dimension $\operatorname{dim} \mathcal{M}(X ; \rho)=0$. One can then consider the element

$$
\phi_{X}=\sum n(X ; \rho)<\rho>.
$$

The numbers $n(X ; \rho)$ can alter as we change the various choice of mode, the basic idea is that the corresponding Floer homology class will not change. Hence one can show that $\partial \psi_{X}=0$ and that the equivalence homology class $\Psi_{X}=$ $\left[\phi_{X}\right] \in H F_{*}(Y)$ is an invariant of $X$, it is called the relative Donaldson invariant.

There is also a corresponding discussion on the cobordism. If $W$ is an oriented cobordism between homology 3 -spheres $Y_{1}$ and $Y_{2}$ there exists an induced map

$$
\Psi_{W: H F_{\imath}}\left(Y_{1}\right) \longrightarrow H F_{\imath+v}\left(Y_{2}\right)
$$

where the grading shift $v=-3\left(b_{2}^{+}(W)-b_{1}\left(W^{r}\right)\right) \bmod 8$ is determined by $W$. Actually we may define a chain map with matrix entries $n\left(\rho_{1}, \rho_{2}\right)$ for $\rho_{1} \in C F_{\imath}\left(Y_{1}\right)$ and $\rho_{2} \in C F_{\imath+v}\left(Y_{2}\right)$, equal to the number of solutions - counted with signs - of a deformed $A S D$ equation on the manifold obtained by adjoining tubes to $W$. These chain-homology maps are independent of the choice of metric.

Our discussion about Floer theory can then be summarized in the following statements, illustrating some features of the Floer homology which is related to 
4-dimensional gauge theory.

- $C F_{*}$ : flat connections over $Y$.

- $\partial$ : 1-dimensional moduli space of $Y \times \mathbb{R}$.

- $\partial^{2}=0$ : 2-dimensional moduli space of $Y \times \mathbb{R}$.

- $\psi_{X}$ or $\phi_{W}$ : 0-dimensional moduli space of $X$ or $W$.

- $\partial \phi_{X}=0$ or $\partial \phi_{W}=0$ : 1-dimensional moduli space of $X$ or $W$.

- $\phi_{X}$ and $\phi_{W}$ being independent of choice of metric modulo $\partial$ : -1 dimensional moduli space over $X$ and $W$.

To complete these preliminaries, in the sequel we discuss the Floer homology groups of more general oriented 3-manifold $Y$, the case is concerned most is where $Y$ has the homology of $S^{1} \times S^{2}$.

Let $P$ be a principal bundle over the oriented 3-manifold $Y$ with the structure group $S O(3)$. Suppose $g$ is an inner automorphism of $P$ (covering identity), we can form a bundle $P_{g}$ over $Y \times S^{1}$ by using $g$ to glue the ends of $\pi_{1}^{*}(P)$ over $Y \times[-\infty,+\infty]$. It is an elementary fact that this sets up a one-to-one correspondence between the isomorphism classes of the bundles over $Y \times S^{1}$, isomorphic to $P$ over $Y \times\{p t\}$, and the connected components of the gauge group $\mathscr{G}(P)$ of $P$ over $Y$. Actually, the fundamental group of the space $\mathscr{B}(P)$ is isomorphic to the set of equivalence classes of bundles over $Y \times S^{1}$. Recall that the $S O(3)$ bundles over $Y \times S^{1}$ are specified by pairs $\left(W_{2}, p_{1}\right)$ with

$$
W_{2}^{2} \cong p_{1}, \bmod 4,
$$

where $W_{2}$ is the second Stiefel-Whitney class, $p_{1}$ is the first Pontrjagin class. The intersection matrix of $Y \times S^{1}$ is even, and this implies that the possible values of $p_{1}$ are just even integers since $W_{2}^{2}=0 \bmod 2$. Thus the 4-dimensional characteristic class over the product detects essential loops in the connection space. It is easy to see that these loops remain essential if we include the reducible connections, so we get a homomorphism

$$
-\frac{1}{2} p_{1}: \pi_{1}(\mathscr{B}(P)) \longrightarrow \mathbb{Z}
$$

Another way to detect the non-trivial loops in $\mathscr{B}(P)$ involves the Chern-Simon functional (cf. [7]). This is a map

$$
\mathscr{C} \&: \mathscr{B}(P) \longrightarrow \mathbb{R} / \mathbb{Z}
$$

which can be defined in various ways. In the preceding discussion of this section, we worked directly on the 3-manifold, and consider for simplicity the case when the bundle $P$ is trivial. Hence we choose a trivialization, so that a connection becomes identified with the Lie algebra valued 1 -form, and set

$$
\mathscr{C} \&(\sigma)=\frac{1}{8 \pi^{2}} \int_{Y} \operatorname{tr}\left(\sigma \wedge d \sigma+\frac{2}{3} \sigma \wedge \sigma \wedge \sigma\right) .
$$


However we now consider the general case where the bundle $P$ may be nontrivial. One can define Chern-Simons up to integers such that the differential of Chern-Simons is the 1 -form $d \mathscr{C} \&$ on $\mathscr{A}(P)$ which assigns to a tangent vector $a \in \Omega^{1}(A d P)$ at a point $\rho \in \mathscr{A}(P)$ the number

$$
d \mathscr{C} \bigotimes_{\rho}(a)=\frac{1}{8 \pi^{2}} \int_{Y}\left(F_{\rho} \wedge a\right)
$$

Then we have

$$
d \mathscr{C} \&_{\rho+b}(a)-d \mathscr{C} \&_{\rho}(a)=\frac{1}{8 \pi^{2}} \int_{Y} \operatorname{tr}\left(d_{\rho} b \wedge b\right)+\mathscr{O}(b)^{2}
$$

by Taylor's expansion. Now we have the identity below

$$
\frac{1}{8 \pi^{2}} \int_{Y} \operatorname{tr}\left(d_{\rho} b \wedge a-b \wedge d_{\rho} a\right)=\frac{1}{8 \pi^{2}} \int_{Y} d \operatorname{tr}(b \wedge a)=0
$$

by Stokes' Theorem, thus the leading term in (1.3) is symmetric in $a, b$ so $d \mathscr{C} \&$ is a closed 1-form. Similarly, by using Stokes' Theorem one sees that $d \mathscr{C} \&$ vanishes on the tangent vectors $a=d_{\rho} \xi$ along the $\mathscr{G}(P)$ orbits, so $d \mathscr{C} \&$ descends to a 1 -form on the quotient $\mathscr{B}^{*}(P)$. Notice that this discussion shows in particular that the critical points, $d \mathscr{b} \&=0$, of the Chern-Simons functional on $\mathscr{B}(P)$ are the gauge equivalence classes of the flat connections on $P$.

It is worth remarking here that the Chern-Simons is the secondary characteristic class. One may fix an oriented 4-manifold $X$ with boundary $Y$, and an extension of the bundle $P$ over $X$ (this can always be done, using the triviality of the cobordism group in 3-manifolds.). Then, for a given connection $\sigma$ on $P$, choose an extension $A$ over $X$, the basic formula for the Chern-Simons [7] is

$$
\mathscr{C} \&(\sigma) \cong \frac{1}{8 \pi^{2}} \int_{X} \operatorname{tr}\left(F_{A} \wedge F_{A}\right) \bmod \mathbb{Z}
$$

In particular, if $W$ is a cobordism between 3-manifolds $Y_{1}$ and $Y_{2}$ and $A$ is a connection over $W$ with boundary value $\sigma_{\imath}$ over $Y_{t}$, then the difference of Chern-Simons over both 3-manifolds $Y_{1}$ and $Y_{2}$ is

$$
\mathscr{C} \&\left(\sigma_{1}\right)-\mathscr{C} \&\left(\sigma_{2}\right)=\frac{1}{8 \pi^{2}} \int_{W} \operatorname{tr}\left(F_{A} \wedge F_{A}\right) \bmod \mathbb{Z} .
$$

Note that the principal $S O(3)$ bundle $P$ can not, in general, be trivialized over $W$ since $P$ has the structure group $S O(3)$. Thus one can not give the natural form of Chern-Simons as in the case where the structure group is $S U(2)$.

Now, considering instantons, we recall the well-known fact that the $A S D$ connection equation $F_{A}+* F_{A}=0$, together with Bianchi identity $d_{A} F_{A}=0$ 


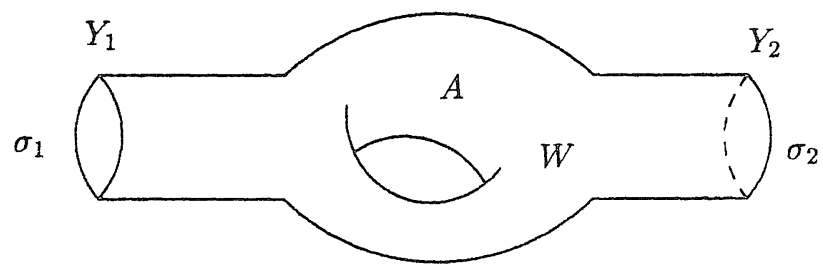

Figure 1

implies that any instanton over the tube $Y \times \mathbb{R}$ satisfies the horizontality condition. So in a temporal gauge instantons can be identified, up to gauge equivalence, with a subset of the paths in $\mathscr{B}(P)$. For such a path in $\mathscr{B}(P)$ to correspond to an instanton is equivalent to it satisfying the differential equation

$$
\frac{d}{d t} A_{t}=*_{3} F_{A v}
$$

where $*_{3}$ is the Hodge star operator on the 3-manifold $Y$. Thus we see that the instanton equation over $Y \times \mathbb{R}$ can be interpreted as the gradient flow equation for the Chern-Simons functional on $\mathscr{B}(P)$.

For every flat connection $\rho \in \mathscr{A}_{\text {flat }}(P)$ there is a chain complex

$$
\Omega^{0}(\mathrm{Ad} P) \stackrel{d_{\bullet}}{\rightarrow} \Omega^{1}(\mathrm{Ad} P) \stackrel{d_{\rho}}{\rightarrow} \Omega^{2}(\mathrm{Ad} P) \stackrel{d_{\bullet}}{\rightarrow} \Omega^{3}(\mathrm{Ad} P)
$$

with associated cohomology groups $H_{\rho}^{\jmath}(Y)$. These cohomology groups can be identified with the spaces of harmonic forms

$$
H_{\rho}^{\prime}\left(Y^{\prime}\right)=\operatorname{Ker} d_{\rho} \cap \operatorname{Ker} d_{\rho}^{*_{3}}
$$

where $d_{\rho}^{* 3}$ denotes the $L^{2}$-adjoint of $d_{\rho}: \Omega^{\prime-1}(\mathrm{Ad} P) \rightarrow \Omega^{\prime}(\mathrm{Ad} P)$ with respect to a Riemannian metric on $Y$. A connection with a discrete isotropy subgroup is called regular, so a regular connection is an irreducible connection. A flat connection is called nondegenerate if $H_{\rho}^{1}(Y)=0$. Note that a flat connection $\rho$ is both regular and nondegenerate if and only if the extended Hessian of Chern-Simons

$$
D_{\rho}=\left(\begin{array}{cc}
*_{3} d_{\rho} & -d_{\rho} \\
d_{\rho}^{*} & 0
\end{array}\right)
$$

is nonsingular (cf. [15], [29]). Here $D_{\rho}$ is a self-adjoint operator on $\Omega^{1}(\mathrm{Ad} P)$ $\oplus \Omega^{0}(\operatorname{Ad} P)$.

We remark here that the condition of every flat connection on $P$ being nondegenerate will in general not be satisfied. If there are degenerate flat 
connections then we perturb the Chern-Simons functional in order to ensure nondegenerate critical points for the perturbed functional (see [6], [15] and [29] for details). Using the sum of the trace of the holonomy along the generators of $H_{1}(Y ; Z)$, Fukaya [19] found a perturbation $f: \mathscr{B}(P) \rightarrow \boldsymbol{R}$, such that the equation with the structure group $S U(2)$

$$
*_{3} F_{\rho}-\operatorname{grad}_{\rho} f=0
$$

has only a finite number of solutions, each of which is nondegenerate. As for the reducibility of flat connections on $P$, we can construct a nontrivial $S O(3)$ bundle over $Y$ such that every flat connection is regular. The proposition below shows which bundles carry no flat reducible connections (cf. [5]).

Proposition 1.1. Let $P \rightarrow Y$ be a principal $S O$ (3)-bundle over an oriented 3-manifold. The following are equivalent:

(1) $P$ carries no flat connection with the holonomy group contained in an $S^{1}$ subgroup of $S O(3)$.

(2) $W_{2}(P) \in H^{2}\left(Y ; \mathbb{Z}_{2}\right)$ does not lift to a torsion class in $H^{2}(Y ; \mathbb{Z})$ under the coefficient map

$$
H^{2}(Y ; \mathbb{Z}) \longrightarrow H^{2}\left(Y ; \mathbb{Z}_{2}\right) .
$$

(3) $W_{2}(P)$ is not zero as a map

$$
H^{2}(Y ; \mathbb{Z}) \longrightarrow \mathbb{Z}_{2}
$$

(4) $W_{2}(P)$ is not zero when evaluated on the fundamental class of an oriented surface $\sum \subset Y$.

Now we consider the gauge transformation group. It is well known that the gauge group is defined by smooth sections of the adjoint bundle, that is, $\mathscr{G}(P)=\Gamma\left(I ; P \times{ }_{\mathrm{Ad}} S O(3)\right)$. Since $S U(2)$ is the double covering of $S O(3)$, we may define

$$
\mathscr{G}_{S}(P)=\Gamma\left(Y^{r} ; P \times{ }_{\mathrm{Ad}} S U(2)\right),
$$

the group $\mathscr{G}_{S}(P)$ is called the restricted group. Thus we get an exact sequence:

$$
\mathscr{G}_{S}(P) \longrightarrow \mathscr{G}(P) \stackrel{\eta}{\longrightarrow} H^{1}\left(Y ; \mathbb{Z}_{2}\right)
$$

where $\eta$ measures the obstruction to deforming a gauge transformation to the identity over the one-skeleton of $Y$. It is easy to show that $\eta$ is onto and that $\mathscr{G}_{S}(P)$ consists of the gauge transformations which lift to $S U(2)$-gauge transformations. Moreover $\pi_{0}\left(\mathscr{G}_{S}(P)\right)=\mathbb{Z}$. Thus the component group of $\mathscr{G}(P)$ now appears as an extension

$$
0 \rightarrow \mathbb{Z} \rightarrow \pi_{0}(\mathscr{G}(P)) \rightarrow H^{1}\left(I^{r} ; \mathbb{Z}_{2}\right) \rightarrow 0
$$


A useful concept of a gauge transformation is its degree. We follow the geometric definition: The degree $d(g)$ of a gauge transformation $g \in \mathscr{G}(P)$ is the intersection number of its graph in $P \times{ }_{\mathrm{Ad}} S O$ (3) with the graph of the identity. The proposition below is due to [6] and [12].

Proposition 1.2. Let $g \in \mathscr{G}(P)$ be such that $\eta(g)$ lifts to an integral class. Then

$$
d(g)=\left(\eta(g) \cup W_{2}(P)\right)[Y] \bmod 2
$$

In general

$$
d(g)=\left(\eta(g) \cup W_{2}(P)+\eta(g)^{3}\right)[\mathrm{Y}] \bmod 2 .
$$

We now return to the Chern-Simons theory. Since the flat connections on $P$ appear as the critical points of the Chern-Simons functional and the 1 -form $d \mathscr{C} \&$ is invariant and horizontal, it follows that the difference $\mathscr{C} \&\left(g^{*} \rho\right)-$ $\mathscr{C} \&(\rho)$ is independent of the connection $\rho$ and is locally independent of the gauge transformation $g$. So it depends only on the component of $\mathscr{G}(P)$, and it turns out that

$$
\mathscr{C} \&\left(g^{*} \rho\right)-\mathscr{C} \&(\rho)=d(g) .
$$

In the remainder of this section, we assume that every flat connection on $P$ is both regular and nondegenerate. According to the preceding discussion about Floer homology for homology 3-spheres, in similar way, we can define Floer homology for the nontrivial $S O(3)$ bundle $P$ over a general 3 -manifold. Fix two flat connections $\rho$ and $\sigma$ on the $S O(3)$ bundle $P$ and consider the space of solutions to the following gradient flow equation:

$$
\frac{d A(t)}{d t}=*_{3} F_{A(t)}
$$

and these solutions also satisfy

$$
\lim _{t \rightarrow-\infty} A(t)=g_{-}^{*} \rho, \quad \lim _{t \rightarrow+\infty} A(t)=g_{+}^{*} \sigma, \quad g_{ \pm} \in \mathscr{G}_{0}(P) .
$$

Where $\mathscr{G}_{0}(P)$ denotes the component of the identity in $\mathscr{G}(P)$, that is,

$$
\mathscr{G}_{0}(P)=\{g \in \mathscr{G}(P) \mid d(g)=0\} .
$$

These solutions are usually termed instantons over $Y \times \mathscr{R}$. The moduli space of these instantons is denoted by

$$
M(\rho, \sigma)=\frac{\{A(t) \in \mathscr{A}(P) \mid(1.7) \text { and }(1.8) \text { are satisfied. }\}}{\mathscr{G}_{0}(P)} .
$$

The next proposition, due to Floer (cf. [15], [17] and Section 2), summarizes 
some key properties of these moduli spaces.

Proposition 1.3. Assume that every flat connection on $P$ is both regular and nondegenerate. For a generic metric on $Y$, the moduli space $M(\rho, \sigma)$ is a finite dimensional oriented paracompact manifold for every pair of flat connections $\rho$ and $\sigma \in \mathscr{A}_{\text {flat }}(\mathrm{P})$. There exists a function $\mu_{P}: \mathscr{A}_{\text {flat }}(P) \rightarrow Z_{\text {such that }}$

$$
\operatorname{dim} M(\rho, \sigma)=\mu(\rho)-\mu(\sigma) \bmod 4 .
$$

This function satisfies

$$
\mu\left(g^{*} \rho\right)-\mu(\rho)=4 d(g)
$$

for $\rho \in \mathscr{A}_{\text {flat }}(P)$ and $g \in \mathscr{G}(P)$.

In terms of flat connections on $P$ and 1-dimensional moduli spaces, we can define the Floer chain complex $C F_{*}$ and the Floer boundary operator $\partial$. It is similar to [15] that the homology of $\left(C F_{*}, \partial\right)$ is an invariant of the bundle $P \rightarrow Y$.

Proposition 1.4. Assume that every flat connection on the SO (3) bundle P over 3-manifold $Y$ is both regular and nondegenerate. Then Floer homology for $P$ on $Y$ is as follows:

(1) The Floer differential $\partial: C F_{*} \rightarrow C F_{*-1}$ satisfies $\partial^{2}=0$. The associated homology gronips

$$
H F_{k}(Y ; P)=\frac{\operatorname{ker} \partial_{k-1}}{\operatorname{Im} \partial_{k}}
$$

are called the Floer homology gronts of the pair $(Y, P)$.

(2) The Floer homology groups are graded modulo 4, if there exists a gauge transformation $g \in \mathscr{G}(\mathrm{P})$ of degree 1 .

(3) The Floer homology groups $H F_{k}(Y ; P)$ are independent of the metric on $Y$ used to construct them.

More details about the instanton homology was discussed in [6], [10]. We devote ourselves to considering the index theory for the $A S D$ equations on the non-compact 4-manifolds in the next section, it is crucial for defining the grading functions of the flat connections over 3-manifolds, and relates the grading shift of the homomorphisms between the instanton homology groups induced by the cobordisms.

\section{§ 2. Index Theory}

We now consider an index theory for the elliptic operators over the 4-manifolds 
with the tubular ends. Thus we wish to associate an "analytical index" to suitable "topological data" (cf. [6], [26], and [30]).

Let $X$ be a 4-manifold with the tubular ends, as defined in Section 1 . The SO (3) bundle $P$ on $X$ is said to be an adapted bundle if it is a smooth bundle with a fixed flat connection over each end. Two adapted bundles are equivalent if there is a bundle isomorphism between them which preserves the flat structures over the ends. Notice that an adapted bundle over $X$ may not be trivial as a $C^{\infty}$ bundle. In general, it is easy to see that the equivalence classes of the adapted bundle with given limits correspond to the invariants of a relative characteristic number. This can be defined by choosing an adapted connection on $P$, i.e., a connection $A$ which agree with the given flat structures over the ends. Then the integral

$$
\frac{1}{8 \pi^{2}} \int_{X} \operatorname{tr}\left(F_{A} \wedge F_{A}\right)
$$

is an invariant of $P$ only.

With those preliminaries in place we proceed to set up our index problem. Let $P$ be an adapted bundle over $X$ with an adapted connection $A$ as above. We assume that each of the limiting flat connections is acyclic. The deformation operator $D=D_{A}$ extends to a Hilbert space to give a bounded operator $D_{A}^{+}: L_{1}^{2} \rightarrow L^{2}$. We also have a formal adjoint operator $\left(D_{A}^{+}\right) *$, and the integration-by-parts formula is valid for sections of the appropriate kind. Notice that the domains of $D_{A}^{+}$and $\left(D_{A}^{+}\right) *$ are quite distinct in general. In a more detailed notations:

$$
\begin{gathered}
D_{A}^{+}: L_{1}^{2}\left(\Omega_{X}^{1}(A d P)\right) \longrightarrow L^{2}\left(\Omega_{X}^{0}(A d P) \oplus \Omega_{X}^{+}(A d P)\right), \\
\left(D_{A}^{+}\right)^{*}: L_{1}^{2}\left(\Omega_{X}^{0}(A d P) \oplus \Omega_{X}^{+}(A d P)\right) \longrightarrow L^{2}\left(\Omega_{X}^{1}(A d P)\right) .
\end{gathered}
$$

Now it need not be true that the operator $D_{A}^{+}$is invertible. We shall see below, that elliptic theory may show that $D_{A}^{+}$is a Fredholm operator. This is just what we would have over a compact base manifold. For compact manifolds one obtains this Fredholm property by piecing together a finite number of inverses defined in local charts, in which the operator is modeled on a constant coefficients operator over Euclidean space [11]. For our case, we have two kinds of models - the familiar Euclidean ones in a system of charts covering a compact interior portion of the manifold and models over the ends (cf. [15], $[26],[28],[30]$ and [31]).

Proposition 2.1. For any adapted connection $A$ over a 4-manifold $X$ with the tubular ends which is flat and acyclic over each end, the operator

$$
D_{A}^{+}: \mathrm{L}_{1}^{2}\left(\Omega_{X}^{1}(A d P)\right) \longrightarrow \mathrm{L}^{2}\left(\Omega_{X}^{0}(A d P) \oplus \Omega_{X}^{+}(A d P)\right)
$$


is Fredholm. That is:

(1) $\operatorname{Ker} D_{A}^{+} \subset L_{1}^{2}\left(\Omega_{X}^{1}(A d P)\right)=\operatorname{Ker} D_{A}^{+} \cap L_{1}^{2}\left(\Omega_{X}^{1}(A d P)\right)$ is finite dimensional.

(2) The image of $D_{A}^{+}$is a closed subspace of finite codimension in $L^{2}\left(\Omega_{X}^{0}(A d P) \oplus \Omega_{X}^{+}(A d P)\right)$.

(3) Moreover, the cokemel $L^{2}\left(\Omega_{X}^{0}(A d P) \oplus \Omega_{X}^{+}(A d P)\right) / \operatorname{Im} D_{A}^{+}$is isomorphic to the kemel of $\left(D_{A}^{+}\right)^{*}$ in $\left.L_{1}^{2}\left(\Omega_{X}^{0}(A d P) \oplus \Omega_{X}^{+}(A d P)\right)\right)$.

We would like to remark here that one may extend the theory to include the adapted bundles over the 4-manifolds whose limits are not acyclic. Thus one has to consider a flat connection $\rho$ over a 3-manifold $Y$ where 0 appears in the spectrum $\{\lambda\}$ of the operator $D_{\rho}$. To recover a Fredholm theory one should use the weighted function spaces. For details, refer to [26], [30].

The index invariant defined in the above has a simple formal property which is basic to Floer's theory. Let $A$ be an adapted connection (with limits) on an $S O$ (3) bundle $P$ over a 4 -manifold $X$ with the tubular ends. Suppose that $X$ contains two boundary components $Y, \bar{Y}$ where $\bar{Y}$ is isomorphic to $Y$ with the reversed orientation; and suppose that the limiting flat connections appearing over $Y$ and $\bar{Y}$ are the same. Consider the family of Riemannian 4 -manifolds $X^{\#(T)}$, depending on a real parameter $T>0$ obtained by identifying the two ends of $X$. Similarly, there is an obvious way of constructing an adapted bundle $P^{\#}$ over $X^{\#(T)}$, using the flat structures to identify the bundles over the endi, and the adapted connection $A$ gives a natural connection $A^{\#}$ on $P^{\#}$. Donaldson et. al. [10] proved that the index of the operator $D_{A \#}^{+}$over $X^{\#(T)}$ is independent of $T$ and the result is written as the following simple formula:

Proposition 2.2. If $X$ and $X^{\#}$ are the 4-manifolds as above. and $P^{\#}$ is obtained from $P$ over $X$ with the acyclic limits via the ghing operation, then

$$
\text { ind }\left(P^{\#}\right)=\text { ind }(P) \text {. }
$$

The most important case of this for us will be when $X$ is disconnected, say a disjoint union of two components $X=X_{1} \cup X_{2}$, and the two ends which are identified are contained in different components of $X$. Then as a direct consequence of Proposition 2.2, one has

Corollary 2.3. In the above situation

$$
\text { ind }\left(P^{\#}\right)=\operatorname{ind}\left(P_{1}\right)+\text { ind }\left(P_{2}\right)
$$

We now consider a particular case when the base manifold is a tube $Y \times \mathbb{R}$. For any a pair of flat connections $\rho, \sigma$ over $Y$ we can define a relative index

$$
\delta_{Y}(\rho, \sigma) \in \mathbb{Z}_{4}
$$


as follows: We choose an $S O(3)$ bundle over the tube $Y \times \mathbb{R}$ with limits $\rho$ at $-\infty$ and $\sigma$ at $+\infty$ respectively, then we can get

$$
\delta_{Y}(\rho, \sigma)=\operatorname{ind}(P) \bmod 4 .
$$

This relative index is a crucial ingredient in Floer's theory - we shall see that this induces the grading in the Floer homology groups. It can be refined slightly to a $\mathscr{Z}_{4}$-grading function $\mu_{P}$ on the acyclic flat connections on the $S O(3)$ bundle $P$ over $Y$. We hope to get an addition relation for the index as follows:

$$
\delta_{Y}(\rho, \sigma)=\mu_{P}(\rho)-\mu_{P}(\sigma) \bmod 4 .
$$

For convenience, here after we denote $\mu_{P}$ by $\mu$ if there is no confusion. Actually, if $P$ is a $S U(2)$ bundle, this definition does not depend on the bundle $P$ used.

The ideas we have been discussing are closely related to the index theory for the manifolds with boundary developed by Atiyah, Patodi and Singer in their series of papers [4]. In particular there are two concepts from this theory - the eta invariant and the spectral flow of a family of elliptic operators. We shall not use the spectral flow in this paper.

We now recall the Atiyah, Patodi and Singer eta invariant. Let $\rho$ be a connection on a SO (3) bundle P over a compact 3-manifold $Y$ and consider the extended Hessian of Chern-Simons:

$$
D_{\rho}=\left(\begin{array}{cc}
*_{3} d_{\rho} & d_{\rho} \\
d_{\rho}^{*} & 0
\end{array}\right): \Omega^{1}(A d P) \oplus \Omega^{0}(A d P) \rightarrow \Omega^{1}(A d P) \oplus \Omega^{0}(A d P) .
$$

This operator is self adjoint with a compact resolvent so its spectrum Spec. $\left(D_{\rho}\right)$ is real and discrete. The series

$$
\eta_{S}\left(D_{\rho}\right)=\sum_{0 \neq \lambda \in \operatorname{Spec} .\left(D_{\rho}\right)} \operatorname{sign} \lambda|\lambda|^{-1}
$$

converges whenever $|s|$ is sufficiently large and defines a meromorphic function of $s$. In [3], [4] it was proved that $s=0$ is not a pole and the eta-invariant of $D_{\rho}$ is defined by

$$
\eta(\rho)=\eta_{0}\left(D_{\rho}\right)
$$

Let $d$ be the corresponding operator on scalar forms and define

$$
\tilde{\eta}(\rho)=\eta(\rho)-3 \eta_{0}(d)
$$

This number $\tilde{\eta}(\rho)$ is defined for every connection on $P$ and is independent of the metric on $Y$ [4]. It does not depend continuously on $\rho$, discontinuities occur whenever 0 is in the spectrum of $D_{\rho}$. However $\tilde{\eta}(\rho)$ is continuous as a function with values in $\mathbb{R} / \mathbb{Z}$.

It is well known that the eta-invariant reflects the asymmetry of the 
spectrum of $D_{\rho}$ and may be thought of as a regularized signature, this fact suggests us to define the grading in Floer homology by using the eta invariant. Now we consider a nondegenerate flat connection $\rho$ on $P$ over $Y$ and define

$$
\mu_{P}(\rho)=2 \mathscr{C} \&(\rho)-\frac{1}{2} \widetilde{\eta}(\rho)+\frac{1}{2} \operatorname{dim} . \quad H^{0}(Y ; A d P)-\frac{3}{2}\left(1+b_{1}(Y)\right)
$$

where

$$
b_{j}(Y)=\operatorname{dim} . \quad H^{j}(Y ; \mathscr{R})
$$

denotes the $j$ th Betti number of $Y$. Each term occurring in the definition of $\mu(\rho)$ is additive with respect to disjoint unions. Thus one has the following lemma (see [6]):

Lemma 2.4. The number $\mu(\rho)$ is an integer and is independent of the metric on $Y$.

We would like to point out here that the integers $\mu(\rho)$ obtained are not well-defined since the definition of $\mu(\rho)$ depends on the choice of ChernSimons, thus the integer $\mu(\rho)$ is only depended up to mod 4 , if $\rho$ is in the gauge equivalence class of flat connections over 3 -manifold i.e. $\mu(\rho) \bmod 4$ is unique. However if the structure group is $S L(2)$, the integer $\mu(\rho)$ defines a natural grading of the instanton homology groups and $\mu(\rho)$ is unique up to mod 8 . Also if the base manifold is a homology $S^{3}$ or homology $S^{1} \times S^{2}$, one may find, for the $S O(3)$ bundle, $\mu(\rho)$ is still the natural grading of the instanton homology groups. In any case, $\mu(\rho)$ gives a relative grading of the instanton homology groups on the $S O(3)$ bundle $P$. The following proposition gives the formula for the dimension of the moduli space over the tube $Y \times \mathbb{R}$, its proof is omitted.

Proposition 2.5. Suppose that $P$ is a non-trivial $S O$ (3) bundle over a 3-manifold $Y$. For every pair of the nondegenerate flat connections, $\rho$ and $\sigma$ on $P$, there exists a generic metric $g$ on $Y$ such that the space $\mathcal{M}_{P, g}(\rho, \sigma)$ is a finite dimensional manifold. Moreover, if $\mathcal{M}_{P, g}(\rho, \sigma)$ is nonempty then

$$
\operatorname{dim} . M_{P, g}(\rho, \sigma)=\mu(\rho)-\mu(\sigma)-\operatorname{dim} \mathscr{G}_{\sigma},
$$

where $\mathscr{G}_{\sigma}$ is the isotropy group of $\sigma$.

Note that the first part of Proposition 1.3 is a direct consequence of the above proposition. For the general case when $X$ is a non-compact 4 -manifold with cylindrical ends the isomorphism class of the triple $(\rho, P, \sigma)$ and the topology of $X$ introduce extra terms in the dimension formula. To describe these we recall that the intersection form on the middle-dimensional cohomology 
group $H^{2}(X ; \mathbb{R})=H^{2}\left(X_{0} ; \mathbb{R}\right)$ is symmetric and is nondegenerate on the image of the homomorphism $H^{2}\left(X_{0}, \partial X_{0} ; \mathbb{R}\right) \rightarrow H^{2}\left(X_{0} ; \mathbb{R}\right)$. Define

$$
\chi^{+}(X)=b_{0}(X)-b_{1}(X)+b_{2}^{+}(X)-b_{0}\left(Y_{2}\right)+b_{1}\left(Y_{1}\right) .
$$

Where $X_{0}$ is a non-compact oriented Riemannian 4-manifold with boundaries $Y_{1}$ and $Y_{2}, X$ is a non-compact oriented Riemannian 4-manifold with the cylindrical ends obtained from $X_{0}$ by adjoining the semi-infinite tubes $Y_{1} \times(-\infty, 0]$ and $Y_{2} \times[0,+\infty)$. More explicitly, we assume that $Y_{1}$ and $Y_{2}$ are compact oriented Riemannian 3-manifolds which contain disjoint components $Y_{12}$. $1 \leq_{i} \leq m$ and $Y_{2 j}, 1 \leq j \leq n$ respectively (see Figure 2 ).

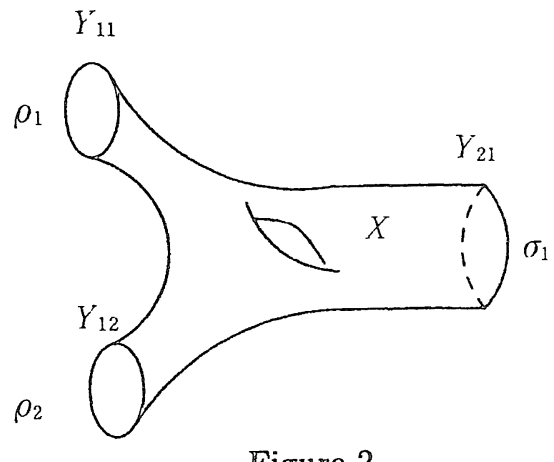

Figure 2

Now suppose that $P$ is an $S O(3)$ bundle over $X$. Let $A$ be an $A S D$ connection on $P$ which has finite energy, and is in the radial gauge as $A$ is restricted to the infinite cylinders $Y_{1} \times(-\infty, 0]$ and $Y_{2} \times[0,+\infty)$. The $A(t)$ converges to $\rho$ and $\sigma$ as $t$ tends to $\pm \infty$ respectively, where $\rho$ and $\sigma$ are disjoint unions of the flat connections $\left(\rho_{1}, \cdots, \rho_{m}\right)$ over ends $\left(Y_{11}, \cdots, Y_{1 m}\right)$, and $\left(\sigma_{1}, \cdots\right.$. $\left.\sigma_{n}\right)$ over ends $\left(Y_{11}, \cdots, Y_{2 n}\right)$ respectively. By Chern-Weil theory, we define the relative characteristic class:

$$
\begin{aligned}
k(\rho, A, \sigma) & =\frac{1}{8 \pi^{2}} \int \operatorname{tr}\left(F_{A} \wedge F_{A}\right)-\mathscr{C} \&(\rho)+\mathscr{C} \&(\sigma) \\
& =\frac{1}{8 \pi^{2}} \int \operatorname{tr}\left(F_{A} \wedge F_{A}\right)-\sum_{i=1}^{m} \mathscr{C} \&\left(\rho_{l}\right)+\sum_{j=1}^{n} \mathscr{C} \&\left(\sigma_{\jmath}\right) .
\end{aligned}
$$

This number is an integer which is a relative Pontrajin number. According to Atiyah-Singer index theory [4], we have the following proposition by using the addition relation for the index:

Proposition 2.6. Under the assumption as discussed above, if $\rho=\left(\rho_{1}, \cdots\right.$, $\left.\rho_{m}\right)$ and $\sigma=\left(\sigma_{1}, \cdots, \sigma_{n}\right)$ are all nondegenerate, then there exists a generic metric $g$ on $X$ such that the moduli space, $\mathcal{M}_{g}^{*}(\rho, A, \sigma)$, for the irreducible ASD connections 
over $X$ is a finite dimensional manifold. Moreover, if $\mathcal{M}_{g}^{*}(\rho, A, \sigma)$ is nonempty then

$$
\operatorname{dim} \mathcal{M}_{g}^{*}(\rho, A, \sigma)=2 k(\rho, A, \sigma)-3 \chi^{+}(X)+\mu(\rho)-\mu(\sigma)-\operatorname{dim} \mathscr{G}_{\sigma}
$$

where

$$
\begin{gathered}
\mu(\rho)=\sum_{\imath=1}^{n} \mu\left(\rho_{\imath}\right), \\
\mu(\sigma)=\sum_{j=1}^{n} \mu\left(\sigma_{\imath}\right), \\
\operatorname{dim} \mathscr{G}_{\sigma}=\sum_{j=1}^{n} \operatorname{dim} \mathscr{G}_{\sigma, .}
\end{gathered}
$$

The proof of the above proposition is given in Appendix $A$. We would like to point out here that:

(1) If the structure group is $S L^{T}(2)$, then the index formula is

$$
\operatorname{dim} M_{g}^{*}(\rho, A, \sigma)=8 k(\rho, A, \sigma)-3 \chi^{+}(X)+\mu(\rho)-\mu(\sigma)-\operatorname{dim} \mathscr{G} \sigma .
$$

where $k(\rho, A, \sigma)$ is the relative second Chern class.

(2) When we consider the self-dual connections over $X$ which is in the radial gauge on the cylindrical ends with $A(t)$ converging to $\rho$ and $\sigma$ as $t$ tends to $-\infty$ and $+\infty$ respectively, then the dimension formula for the self-dual moduli space is

$$
\operatorname{dim} \mathcal{M}_{g}^{*}(\rho, A, \sigma)=2 k(\rho, A, \sigma)-3 \chi^{-}(X)+\mu(\rho)-\mu(\sigma)-\operatorname{dim} \mathscr{G}_{\rho} .
$$

where

$$
\chi^{-}(X)=b_{0}(X)-b_{1}(X)+b_{2}^{-}(X)-b_{0}\left(Y_{1}^{r}\right)+b_{1}\left(Y_{2}\right)
$$

and

$$
k(\rho, A, \sigma)=\frac{-1}{8 \pi^{2}} \int_{X} \operatorname{tr}\left(F_{A} \wedge F_{A}\right)+\mathscr{C} \&(\rho)-\mathscr{C} \&(\sigma)
$$

Note that here $k(\rho, A, \sigma)$ is the relative first Pontrajin number. The ChernSimons, for this case, is given as follows: For a given connection $\rho$ on the $S O(3)$ bundle $P$ over the 3 -manifold $Y$, choose an extension $A$ over $X$ which is an oriented 4-manifold with boundary $Y$, the basic formula for the Chern-Simons invariant is

$$
\mathscr{C} \&(\rho) \cong \frac{1}{8 \pi^{2}} \int_{X} \operatorname{tr}\left(F_{A} \wedge F_{A}\right) \bmod \mathbb{Z}
$$

Hence the grading function $\mu$ is similar to original $A S D$ case:

$$
\mu(\rho)=2 \mathscr{C} \&(\rho)-\frac{1}{2} \tilde{\eta}(\rho)+\frac{1}{2} \operatorname{dim} . H^{0}\left(Y^{r} ; A d P\right)-\frac{3}{2}\left(1+b_{1}(Y)\right) .
$$




\section{$\S 3 . \quad$ Dehn Surgery and Cobordism Functors}

In this section, we devote ourselves to discussing Dehn surgery on a knot in a 3-manifold, and the cobordism functors induced by that surgery. First we digress to consider the instanton homology on an $S O(3)$ bundle over a 3-manifold.

Suppose that $Y$ is an oriented, compact 3 -manifold, $P$ is a non-trivial SO (3) bundle over the 3-manifold $Y$ that means that $P$ has non-zero the second Stiefel-Whitney class $W_{2}(P) \neq 0$. Suppose that every flat connection on $P$ is both regular and non-degenerate. As in the case whose structure group is $S U(2)$, let $R(Y)$ denote the critical point set of Chern-Simons with equivalencc, then $R(Y)$ becomes the set of the simplexes in the instanton homology. Similarly, using the index formulae discussed in the last section, one may define the grading $\mu: R(Y) \rightarrow \mathbb{Z}$ which is unique up to $\bmod 4$. Hence for any two flat connections $\rho, \sigma \in R(Y)$, the virtual dimension of $\mathcal{M}(\rho, \sigma)$ over the tube $Y \times \mathbb{R}$ is equal to the relative index ind $(\rho, \sigma)$ :

$$
\operatorname{dim} . M(\rho, \sigma)=\mu(\rho)-\mu(\sigma) \bmod 4 .
$$

Therefore one can define a boundary operator $\partial$ of the instanton homology on $P$ by using the one dimensional moduli space over the tube $Y \times \mathscr{R}$. Thus one can obtain the instanton homology on an $S O(3)$ bundle $P$ graded by $\mathbb{Z}_{4}$. It is independent of the choice of metric on $Y$ and perturbations.

These instanton homology groups allow one to extend the Donaldson's invariants for the closed 4-manifold to the non-compact 4-manifold with the boundary. In particular, an oriented cobordism between two 3-manifolds induces a homomorphism of the instanton homology groups over those two 3-manifolds. Suppose that $W$ is an oriented cobordism between 3-manifolds $Y_{1}$ and $Y_{2}$, and $P$ is a non-trivial $S O(3)$ bundle over $W$. Let $P_{1}, P_{2}$ denote $P_{1}=$ $\left.P\right|_{Y_{1}}, P_{2}=\left.P\right|_{Y_{2}}$ respectively, then $W$ induces maps:

$$
\Psi_{W:} H F_{i}\left(P_{1}\right) \longrightarrow H F_{i+v}\left(P_{2}\right),
$$

where the grading shift

$$
\nu=2 k-3 \chi^{+}(W) \bmod 4
$$

and where

$$
\chi^{+}(W)=b_{0}(W)-b_{1}(W)+b_{2}^{+}(W)-b_{0}\left(Y_{2}\right)+b_{1}\left(Y_{1}\right) .
$$

and the integer $k$ is the relative Pontrjagin class of $P$. If $W$ is a cobordism between two homology 3 -spheres, then the grading shift is

$$
\nu=2 k+3\left(b_{1}(W)-b_{2}^{+}(W)\right) \bmod 4 .
$$

When one chooses an $S U(2)$ bundle, then $k$ becomes a relative Chern class. thus 
the grading shift is

$$
\nu=3\left(b_{1}(W)-b_{2}^{+}(W)\right) \bmod 8 .
$$

Recall that the homomorphism $\Psi_{W}$ is defined by the 0 -dimensional moduli space on $P$. It is easy to show that the formulae for the grading shift are direct consequence of the index theory developed in $\S 2$.

These maps induced from the cobordism enjoy the functorial properties. which are manifestations of the "gluing" of the instanton solutions. We would like to point out several properties of the cobordism functors that we shall encounter again later (cf. [10], [15]). For each $(W, P)$ as described above, the induced map

$$
\Psi_{W:} H F_{*}\left(P_{1}\right) \longrightarrow H F_{*}\left(P_{2}\right)
$$

depends only on the smooth cobordism $W$ and the $S O(3)$ bundle. It is the identity for the product cobordism. For a composite cobordism $W=U V$, if the $S O$ (3) bundles $P_{U} \rightarrow U$ and $P_{V} \rightarrow V$ are the restrictions of the $S O$ (3) bundle $P \rightarrow$ $W$ to $U$ and $V$ respectively, then $\Psi_{W}=\Psi_{U}{ }^{\circ} \Psi_{V}$.

We now discuss Dehn surgery and the cobordism functors induced from that surgery. It plays an important role in our exactness theorem for the instanton homology groups on an $S O(3)$ bundle over an oriented compact 3-manifold.

Recall the Dehn construction. Surgery in general is the process of removing a solid torus, $N \cong S^{1} \times D$, from a 3 -manifold $Y$, and identifying the boundary of the hole with the boundary of another solid torus, $N^{\prime}$, via a homeomorphism different from the one defined by the inclusion on $N$ in $Y$ (often called sewing $N$ back differently).

Now suppose that $Y$ is an oriented homology 3 -sphere and $\mathscr{K} \subset Y$ is a knot: a smooth embedded circle in $Y$. One may introduce two other 3-manifolds by Dehn surgery:

(1) The homology 3 -sphere $Y^{\prime}$ obtained from $Y$ by +1 surgery on $\mathscr{K}$.

(2) The homology $S^{1} \times S^{2} Y^{\prime \prime}$ obtained from $Y$ by 0 surgery on $\mathscr{K}$.

The definitions here are standard in 3-manifold topology [21], [22]. More specifically, let $N$ be a tubular neighborhood of $\mathscr{K}$ in $Y$ and

$$
\varphi: S^{1} \times S^{1} \longrightarrow \partial N
$$

be the natural identification fixed so that $\varphi\left(S^{1} \times p t\right.$. $)$ maps to zero in $H_{1}(N)$ and $\varphi\left(p t . \times S^{1}\right)$ maps to zero in $H_{1}(Y \backslash N)$ (Thus the first generator is the "meridian" and the second is the "longitude" of the knot). The 3-manifold $Y^{\prime \prime}$ is obtained by cutting out $N$ and gluing it back using the diffeomorphism of $S^{1} \times S^{1}$ which interchanges the two factors: in the homology the situation is the same as going from $S^{3}$ to $S^{1} \times S^{2}$ by regluing two $S^{1} \times S^{1}$ s. That is 


$$
Y^{\prime \prime}=N \underset{\xi_{0}}{\bigcup}(Y \backslash N)
$$

where $\xi_{0}: \partial N \rightarrow \partial(Y \backslash N)$ is the conjugate by $\varphi$ of the diffeomorphism

$$
(\theta, \Phi) \mapsto(\Phi, \theta)
$$

of the torus, where $\langle\theta, \Phi)$ is the coordinate of $S^{1} \times S^{1}$. The 3 -manifold $Y^{\prime}$ is obtained similarly as

$$
Y^{\prime}=N \underset{\xi+1}{\bigcup}(Y \backslash N)
$$

where $\xi_{+1}: \partial N \rightarrow \partial(Y \backslash N)$ is the conjugate by $\varphi$ of the diffeomorphism

$$
(\theta, \Phi) \mapsto(\theta-\Phi, \Phi)
$$

of the torus. We remark here that the 3-manifold $Y$ can be regarded as

$$
Y=N \underset{\imath d .}{\cup}(Y \backslash N) \text {. }
$$

There are standard, oriented, surgery cobordisms $W_{1}$ from $Y^{\prime}$ to $Y$ and $W_{2}$ from $Y$ to $Y^{\prime \prime}$ which are illustrated in the following diagram:

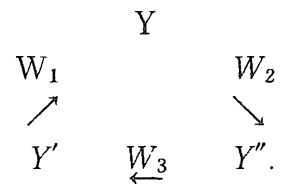

In particular, if we let $W$ be the composite cobordism from $Y^{\prime}$ to $Y^{\prime \prime}$, there is an embedded 2-sphere in $W^{r}$ with self-intersection -1 according to our construction. This self-intersection number fixes the sign $(+1$ surgery $)$ in the set-up, and it is crucial that we consider this sign rather than the opposite: +1 surgery on $Y$ yields in general a different manifold to -1 surgery. Also, we may define another cobordism $W_{3}$ from $Y^{\prime \prime}$ to $Y^{\prime}$ as in the above diagram. In fact $W_{3}^{r}$ is the manifold obtained from $\bar{W}$ by collapsing or "blowing down" the embedded 2-sphere where $\bar{W}$ is the composite cobordism $W_{2} W_{1}$ with reversed orientation. Hence $\bar{W}=W_{3} \# \mathbb{C} P^{2}$. In terms of the definition of $W_{3}$, one has that $H^{*}\left(W_{3}\right) \cong H^{*}\left(B^{2} \times S^{2} \backslash B_{1}^{4}\right)$, therefore $b_{1}\left(W_{3}\right)=0, b_{2}^{+}\left(W_{3}\right)=0$, moreover $b_{2}\left(W_{3}\right)=1$.

To calculate the grading shifts of the cobordisms, we digress to consider the intersection forms and the homology groups of the cobordisms. We have $Y^{\prime}$ and $Y^{\prime \prime}$ obtained from +1 surgery and 0 surgery on a knot $\mathscr{K}$ in a homology 3 -sphere $Y$. By the assumption, $W_{1}$ and $W_{2}$ are the cobordisms from $Y^{\prime}$ to $Y$ and $Y$ to $Y^{\prime \prime}$ respectively. Let us try to visualize the 2-homology of $W_{1}$ and $W_{2}$.

Let $F$ be a Seifert surface of $\mathscr{K}$ in $Y$. Let $D_{1}$ and $D_{2}$ be the core 2-disk of the 2-handles attached to $Y$ to obtain $W_{1}$ and $W_{2}$ respectively. Then $H_{2}\left(W_{1}\right)$ is 
generated by $F+D_{1}$ with self-intersection -1 ; and $H_{2}\left(W_{2}\right)$ is generated by $F+D_{2}$ with self-intersection 0 . So, since $Y$ is a homology 3 -sphere, $H_{2}\left(W_{2} W_{1}\right)$ is generated by $F+D_{1}$ and $F+D_{2}$. The difference of these two homology classes is represented by an embedded 2-sphere $D_{2}-D_{1}$ with self-intersection - 1. So one can split out one $\overline{\boldsymbol{C P}}^{2}$ (or $\boldsymbol{C P} P^{2}$ if one reverses the orientation of $\left.W_{2} W_{1}\right)$. As far as $H_{2}$ is concerned, what is left can be imaged as two copies of $F$ glued together along their boundary (the knot $\mathscr{K}$ embedded in $Y$ ) with intersection -1 . According to the definition of $W_{3}$, we claim that $W_{3}$ looks like $S^{2} \times D$ homologically. Notice that $W_{3}$ is a cobordism from $Y^{\prime \prime}$ to $Y^{\prime}$.

A few remarks are in order. According to our construction, the cobordism $W_{1}$ has $b_{1}\left(W_{1}\right)=b_{2}^{+}\left(W_{1}\right)=0$, where as $b_{2}^{-}\left(W_{1}\right)=1$ with a class represented by the disk, $D_{1}$, capped off by a surface (Seifert surface $F$ ) in $Y$. Thus, $W_{1}$ looks like $\overline{C P}^{2}$ homologically. Similarly, $W_{2}$ looks like $S^{2} \times D$ homologically, thus $b_{1}\left(W_{2}\right)=b_{2}^{+}\left(W_{2}\right)=b_{2}^{-}\left(W_{2}\right)=0$, but $b_{2}\left(W_{2}\right)=1$ with a class represented by the disk, $D_{2}$, capped off by Seifert surface $F$ in $Y$. Finally, $W_{3}$ also looks like $S^{2} \times D$ homologically, we find that $b_{1}\left(W_{3}\right)=b_{2}^{+}\left(W_{3}\right)=0, b_{2}\left(W_{3}\right)=1$.

We need the following lemma which relates the cobordism functors to be discussed shortly.

Lemma 3.1. Suppose that $W_{\mathbf{L}}, W_{2}, W_{3}$ are the cobordisms from $Y^{\prime}$ to $Y$, from $Y$ to $Y^{\prime \prime}$ and from $Y^{\prime \prime}$ to $Y^{\prime \prime}$ respectively as in the preceding discussion. Then the composite $W_{3} W_{2} W_{1}$ is also a cobordism from $Y^{\prime \prime}$ to $Y^{\prime}$, it looks like $\overline{\mathrm{CP}}^{2} \# \mathbb{C P}^{2} \# \overline{\mathrm{CP}}^{2}$ homologically.

Proof. According to the above discussion, the composite $W_{2} W_{1}$ looks like $\bar{W}_{3} \# \overline{C P}^{2}$ homologically, here $\bar{W}_{3}$ is the $W_{3}$ with the reversed orientation. Thus $W_{3} \bar{W}_{3}$ looks like $S^{2} \times S^{2}$ homologically since $W_{3} \bar{W}_{3}$ is a double covering of $W_{3}$ along the homology $S^{1} \times S^{2} Y^{\prime \prime}$. Hence one may "slide a 2-handle over a 1-handle" [22], it follows that $W_{3} W_{2} W_{1}$ looks like $S^{2} \times S^{2} \# \overline{C P}^{2} \cong \overline{C P}^{2} \# \mathbb{C} P^{2} \#$ $\overline{C P}$. The proof is complete.

Using Lemma 3.1 and the index formulae (Section 2), by the index calculation, we can show that the surgery triangle of the knot $\mathscr{K}$ in the homology 3 -sphere $Y$ is the triple

$$
H F_{*}\left(Y^{\prime}\right) \stackrel{a_{*}}{\rightarrow} H F_{*}(Y) \stackrel{0_{*}}{\rightarrow} H F_{*}\left(Y^{\prime \prime}\right) \stackrel{\prime_{*}^{*}}{\rightarrow} H F_{*}\left(Y^{\prime}\right)
$$

of the surgery cobordisms, where the second Stiefel-Whitney class is trivial relative to the boundaries and with the ends identified in such a way that its total degree is -1 . 
Proposition 3.2. Suppose that as above, $a_{*}, b_{*}$ and $c *$ are the homomorphisms of the Floer homology groups induced by the cobordism functors. Then $a_{*}$ and $b_{*}$ have degree 0 with respect to the grading, and $c_{*}$ has degree -1 with respect to the grading. Hence the surgery triangle of $\mathscr{K}$ is the triple

$$
H F_{*}\left(Y^{\prime}\right) \stackrel{a_{*}}{\rightarrow} H F_{*}(Y) \stackrel{b_{*}}{\rightarrow} H F_{*}\left(Y^{\prime \prime}\right) \stackrel{c_{*}}{\rightarrow} H F_{*-1}\left(Y^{\prime}\right)
$$

Proof. First let us consider the degree of $c_{*} b_{*} a_{*}$. By Lemma 1, the composite $W_{3} W_{2} W_{1}$ is a cobordism from $Y^{\prime}$ to $Y^{\prime}$, it looks like $\overline{\mathbb{C} P}^{2} \# \mathbb{C} P^{2} \# \overline{\mathbb{C} P}^{2}$ homologically, so $b_{2}^{+}\left(W_{3} W_{2} W_{1}\right)=1$. Note that $Y^{\prime}$ is a homology 3 -sphere. According our construction of Dehn surgery, the second Stiefel-Whitney class on the $S O(3)$-bundle over $W_{3} W_{2} W_{1}$ should be non-trivial, and the relative. Pontrjagin class $p_{1}$ should be odd modulo 4 . Hence using the index formula, the shift of the grading induced by the composite $W_{3} W_{2} W_{1}$ is

$$
\nu=2 k\left(W_{3} W_{2} W_{1}\right)-3 \chi^{+}\left(W_{3} W_{2} W_{1}\right)=2-3=-1 \bmod 4 .
$$

On the other hand, the shift of the grading induced by the cobordisms $W_{1}$ and $W_{2}$ are always even. So the degree of $a_{*}, b_{*}$ are zero modulo 4 , the degree of $c_{*}$ must be -1 modulo 4 . The proof is complete.

Finally, we would like to point out that the extension of the framed surgery cobordism to the $S O(3)$-bundle is unique up to the second Stiefel-Whitney class between the ends. That the total degree of the composite cobordisms is equal to -1 modulo 4 follows from an index calculation. The construction becomes perfectly symmetric in the three maps in (3.6) if one formulates the surgery problems for general knots. The details are in [6], [16], [17].

\section{§4. Morse-Smale Type $\mathbb{F}$ low for $\mathbb{K}$ mots}

In this section, we give a brief discussion on the deformation equations and Hamiltonians of the knots embedded in 3-maifolds. For more details, see [6], [16]. [17] and [18].

Let $Y$ be a homology 3 -sphere, $\mathscr{K}$ be a knot embedded in $Y$. By the +1 -surgery and 0 -surgery on the knot $\mathscr{K}$, we obtain another homology 3 -sphere $Y^{\prime}$ and a homology $S^{1} \times S^{2} Y^{\prime \prime}$ respectively. There are standard surgery cobordisms $W_{1}$ from $Y^{\prime}$ to $Y$ and $W_{2}$ from $Y$ to $Y^{\prime \prime}$ respectively.

To obtain an exactness theorem for the Floer homology groups of the manifolds $Y^{\prime}, Y, Y^{\prime \prime}$, we define certain perturbations of the Chern-Simons which "simulate" the effect of the surgery. To do this, we may consider the perturbations defined by the holonomy of connections around the knot $\mathscr{K}$. More precisely, fix a smooth compactly support volume 2 -form $\mu$ on a disc $D$, of 
integral 1, choose a suitable function $\phi$ from the structure group $S U$ (2) (or $S O(3))$ to the real line $\mathscr{R}$ which is invariant under the adjoint action of $S U(2)$ (or $S O(3)$ ). Fix coordinates to identify a neighborhood of $\mathscr{K}$ with $\mathscr{K} \times D$, and write $\mathscr{K}_{z}$ for the obvious loop, parallel to $\mathscr{K}$ by a point $z \in D$. Now for each fixed $z$ we can construct a function $\Phi_{z}$ on the space of the connections, in the following manner:

Let $P$ be an $S U(2)$ bundle over $Y$, and $T\left(A, \mathscr{K}_{z}\right)$ be the holonomy of $A$ around $\mathscr{K}_{z}$, which can be regarded as an automorphism of the fiber of $P$ over any point of $\mathscr{K}_{z}$. The holonomy yields a conjugacy class in $S U(2)$, so it makes sense to define a real number

$$
\Phi_{z}=\phi\left(T_{A}, \mathscr{K}_{z}\right)
$$

Then we put

$$
\Phi(A)=\int_{D} \Phi_{z} \mu(z)
$$

We can read off the derivative of $\Phi$ (cf. [6]) by linearity:

$$
\delta \Phi=-\int_{Y} \operatorname{tr}\left(a \phi^{\prime}\left(T_{A}\right)\right) \wedge \mu
$$

Here $T_{A}$ is the section of $P \times_{a d} S U(2)$ over the support of $\mu$ defined by using the holonomy around $\mathscr{K}_{z}$ at a point on $\mathscr{K}_{z}$.

Thus the derivative of the perturbed Chern-Simons functional, $\mathscr{C} \&+\Phi$, on the space of the connections is

$$
\delta(\mathscr{C} \&+\Phi)=\int_{Y} \operatorname{tr}\left(a \wedge\left(F_{A}-\phi^{\prime}\left(T_{A}\right) \mu\right)\right.
$$

and the critical points of $\mathscr{C} \&+\Phi$ the analogue of flat connections in the deformation theory, are the solutions $A$ of the equation

$$
F_{A}=\phi^{\prime}\left(T_{A}\right) \text {. }
$$

Let $Y_{0}$ be the common knot complement, since $Y$ is a homology 3-sphere we may make a generic auxiliary perturbation in the interior of $Y_{0}$ such that, apart from the trivial connection, reducible connections do not enter when we study the flat connections over $Y^{\prime}, Y, Y^{\prime \prime}$. Simultaneously, for each irreducible flat connection $\rho$ over the knot-complement $Y_{0}$ : (1) the restriction of $\rho$ to the torus $\partial(\mathscr{K} \times D)$ does not have the holonomy contained in the center \pm 1 of $S U(2)$; (2) the cohomology group $H^{2}\left(Y_{0}, a d \rho\right)$ is zero, so the space of the equivalence classes of the representations of the knot complement, $R_{Y_{0}}$, is of dimension 1 ; (3) $R_{Y_{0}}$ is transverse to any of the three submanifolds $\operatorname{int} L_{Y^{\prime}}, \operatorname{int} L_{y}, \operatorname{int} L_{Y^{\prime \prime}}$ at $\rho$, where $L_{Y^{\prime}}, L_{Y}, L_{Y^{\prime \prime}}$ are three lines in the dual torus of $\partial(\mathscr{K} \times D)$ which correspond to the three 3 -manifolds $Y^{\prime}, Y$ and $Y^{\prime \prime}$. Then we can choose a 
suitable perturbation $\Phi$ supported in the neighborhood $\mathscr{K} \times D$ such that the set $\widetilde{R}$ of the irreducible critical points of $\mathscr{C} \mathscr{\&}+\Phi$ is identified, under the restriction to the common knot complement $Y_{0}$, with the union

$$
\widetilde{R}=\mathscr{R}_{Y^{\prime}} \cup \mathscr{R}_{Y^{\prime \prime}} .
$$

Hence we conclude that there is a chain complex $\left(\widetilde{C F}_{*}, \widetilde{\partial}\right)$ with the chain groups generated by the set $\widetilde{\mathscr{R}}$, and the differential is defined by the gradient curves of $\mathscr{C} \&+\Phi$ - the deformed-instanton over the tube - and the homology of this chain complex is canonically isomorphic to the Floer homology $H F_{*}(Y)$. By the construction, the chain group $\widetilde{C F}_{*}(Y)$ is identified as a group with the direct sum

$$
\overline{C F}_{*}(Y)=C F_{*}\left(Y^{\prime}\right) \oplus C F_{*}\left(Y^{\prime \prime}\right) .
$$

To obtain the chain maps

$$
C F *\left(Y^{\prime}\right) \longrightarrow \overrightarrow{C F}_{*}(Y) \longrightarrow C F\left(Y^{\prime \prime}\right),
$$

consider the deformed instanton equation over the cobordisms $W_{1}$ and $W_{2}$. We should choose the time-dependent perturbations of the instanton equation over $W_{1}$ and $W_{2}$. compatible with the given data on the boundaries. As in the undeformed case that the zero-dimensional moduli spaces of the time-dependent perturbed instanton equation over the cobordisms $W_{1}, W_{2}$ induce the chain maps $\tilde{a}$ and $\tilde{b}$ from $C F *\left(Y^{\prime}\right)$ to $\widetilde{C F}_{*}(Y)$, and from $\widetilde{C F}_{*}(Y)$ to $C F *\left(Y^{\prime \prime}\right)$ respectively, also these induce the same maps $a_{*}$ and $b *$ on the Floer homology. Note that $\left(\widetilde{C F}_{*}(Y), \widetilde{\partial}\right)$ is defined by the perturbed Chern-Simons functional, hence $\tilde{a}$ and $\tilde{b}$ are homotopic to $a$ and $b$ respectively.

Since the isomorphism (4.5) is an isomorphism of the chain complexes, the chain maps $\tilde{a}, \tilde{b}$ could be similar to the inclusion $i: C F_{*}\left(Y^{\prime}\right) \rightarrow C F_{*}\left(Y^{\prime \prime}\right) \oplus C F_{*}$ $\left(Y^{\prime \prime}\right)$, and the projection $\pi: C F_{*}\left(Y^{\prime}\right) \oplus C F_{*}\left(Y^{\prime \prime}\right) \rightarrow C F_{*}\left(Y^{\prime \prime}\right)$ respectively. To get this, one needs the "monotonicity" property of the cobordism functor with respect to the filtration of the Floer groups. i.e., the map induced by the cobordism decreases the Chern-Simon functional. It is well known that an $S U(2)$ bundle over a closed 4-manifold which admits an instanton connection must have positive second Chern class. This follows from the fact that the Chern-Weil integrand $\operatorname{tr}(F \wedge F)$ is equal to $|F|^{2}$ for an $A S D$ connection. Hence the map induced by the cobordism and the Floer differential will decrease the Chern-Simons functional. The decreasing property means that we have a filtered complex, so there exists a corresponding intrinsic filtration of the Floer groups and the Floer homology theory has a filtration which is natural with respect to the cobordism functor. 
As we saw, the "exact triangle" is due to the basic point that any side can be deformed into the union of the other two. Hence we use the perturbations to simulate the effect of surgery, the maps induced by the corresponding cobordisms are the solutions of the time-dependent deformation. But those maps given by the general time-dependent deformations are not compatible with the filtration. To overcome this difficulty, we should introduce the timeindependent deformations since the whole discussion on the undeformed equation can go over to the case of a time-independent deformation of the instanton equations: The Floer chain groups for the deformed functionals $\mathscr{C} \&+\Phi$ at each end have the filtration and the chain map defined by the solutions over the cobordism is decreasing with respect to these filtration [6].

Proposition 4.1. Let $\tilde{a}, \tilde{b}$ be the chain maps between the filtered complexes in (4.5). There are the deforned instanton equations over the cobordisms $W_{1}, W_{2}$ whose chain maps satisfy the following conditions with respect to the filtration of the complexes $C F_{*}\left(Y^{\prime}\right), \overline{C F}_{*}(Y), C F_{*}\left(Y^{\prime \prime}\right)$ :

(1) $\tilde{a}=i+\alpha, \tilde{b}=\pi+\beta$, where $\alpha, \beta$ are strictly decreasing with respect to the filtration, and $i, \pi$ form a short exact sequence of group homomorphisms.

(2) The composite $\tilde{b} \widetilde{a}$ is chain homotopic to zero by a homotopy $H: C F *\left(Y^{\prime}\right) \rightarrow$ $C F_{*}\left(Y^{\prime \prime}\right)$ (i.e. $\left.\partial H+H \partial=\widetilde{\mathrm{b}} \widetilde{\mathrm{a}}\right)$, where $H$ is strictly decreasing with respect to the filtration.

(3) There is a long exact homology sequence,

$$
\cdots \rightarrow H F_{*}\left(Y^{\prime}\right) \stackrel{a_{*}}{\rightarrow} * H F_{*}(Y) \stackrel{b_{+}}{\rightarrow} H F_{*}\left(Y^{\prime \prime}\right) \stackrel{\Delta_{*}}{\rightarrow} H F_{*-1}\left(Y^{\prime}\right),
$$

and in particular the homomorphism $\triangle_{*}$ is induced by the bonndary operator $\partial$.

It is worth remarking here that the time independent deformations of the instanton equations give the decreasing maps, with respect to the filtration, but it is clear that we can not use such deformations directly, since on each of $W_{1}$, $W_{2}$ the problem is only perturbed at one end. The master-stroke in the proof of Proposition 4.1 is to puncture the manifolds $W_{1}, W_{2}$ to obtain manifolds $\widehat{W}_{1}$, $\widehat{W}_{2}{ }_{2}$ with an additional end, and then to compare the time-dependent deformations on the $W_{t}$ with the time-independent deformations on the $\widehat{W}_{t}$ (cf. [6]).

In the next section, we study the homomorphism $\Delta_{*}$ induced by the boundary operator $\partial$. By using Morse type flow of knots, we prove that the homomorphism $\Delta_{*}$ coincides with the homomorphism $c *$ induced by the cobordism $W_{3}$, and complete the proof of the main theorem. 


\section{$\S 5$. Homomorphism $\triangle_{*}$ Induced by Boundary Operator $\partial$}

In this section, we study the homomorphism $\Delta_{*}$ induced by the boundary operator $\partial$ and construct a cobordism such that the corresponding homomorphism due to that cobordism is isomorphic to $\Delta_{*}$. Ultimately, we prove that the homomorphism $\Delta_{*}$ is equivalent to the homomorphism $c_{*}$ induced by the cobordism $W_{3}$ and complete the proof of the main theorem. We use an idea similar to the vanishing argument used in [11], [23] and [24].

First, recall the construction of the homomorphism $\Delta_{*}$. We may puncture the manifolds $W_{1}, W_{2}$ to obtain the manifolds $\widehat{W}_{1}, \widehat{W}_{2}$ with an additional end, and then compare the time-dependent deformations on the $W_{\imath}(i=1,2)$ with the time-independent deformations on the $\widehat{W}_{i}$. Let $\widehat{W}_{i}$ be the manifolds obtained. from $W_{i}$ by removing an interior point, which we may take to lie on the disc $D_{\imath}$ (cf. Sect.3), and with the metric giving the manifold an additional tubular end modeled on $S^{3} \times \mathscr{R}$. We can find a local product region in $\widehat{W}_{1}$ which interpolates between the neighborhood of $\mathscr{K} \in Y$ and the neighborhood of the standard circle in $S^{3}$. Introduce the time-independent deformations on $\widehat{W}_{i}$. Then $W_{\imath}$ can be regarded as the connected sum of $\widehat{W}_{\imath}$ and $Q_{\imath}$ across $S^{3}$, where $Q_{l}$ is the 4-ball with a metric having one tubular end. By using the monotonicity of the time-independent deformations on $\widehat{W}_{l}$, we get the chain maps

$$
C F_{*}\left(Y^{\prime}\right) \stackrel{\tilde{a}}{\rightarrow} \widetilde{C F} *(Y) \stackrel{\vec{b}}{\rightarrow} C F *\left(Y^{\prime \prime}\right),
$$

where $\widetilde{a}=i+\alpha . \tilde{b}=\pi+\beta, \alpha$ and $\beta$ are strictly decreasing with respect to the filtration, and $i, \pi$ form a short exact sequence of group homomorphisms [6]. It is easy to see that there is a right inverse, say $R$, to $\widetilde{b}$ (we shall give more details shortly). Thus we put $K=R H$, where $H$ is a homotopy by which the composite $\tilde{b} \widetilde{a}$ is chain homotopic to zero (i.e. $\partial H+H \partial=\tilde{b} \widetilde{a}$ ).

Then

$$
\widetilde{b}(\partial K+K \partial)=\partial \widetilde{b} R H+\widetilde{b} R H \partial=\partial H+H \partial=\widetilde{b} \widetilde{a},
$$

so

$$
\tilde{b}(\widetilde{a}-(\partial K+K \partial))=0 .
$$

Note that $\tilde{b} \partial=\partial \tilde{b}$ since the homomorphism $\tilde{b}$ is induced by the cobordism $W_{2}$. We define $\widetilde{a}_{1}$ to be $\widetilde{a}-(\partial K+K \partial)$. Then we get a short exact sequence

$$
0 \rightarrow C F_{*}\left(Y^{\prime}\right) \stackrel{\tilde{a}_{1}}{\rightarrow} \widetilde{C F} *(Y) \stackrel{\tilde{b}}{\rightarrow} C F_{*}\left(Y^{\prime \prime}\right) \rightarrow 0 .
$$


Since $\tilde{a}$ is chain homotopic to $\tilde{a}_{1}$, by $(5.2)$ we get a long exact homology sequence,

$$
\cdots \rightarrow H F_{*}\left(Y^{\prime}\right) \stackrel{a_{*}}{\rightarrow} \widetilde{H F} *(Y) \stackrel{b_{+}}{\rightarrow} H F_{*}\left(Y^{\prime \prime}\right) \stackrel{\Delta_{x}}{\rightarrow} H F_{*-1}\left(Y^{\prime}\right) \rightarrow \cdots
$$

where $\widetilde{H F}_{*}(Y) \cong H F_{*}(Y)$ and $\triangle_{*}$ is induced by the boundary operator $\partial$.

To compare $\Delta_{*}$ with $c_{*}$ induced by the cobordism $W_{3}$, let us define $\Delta_{*}$ by using the short exact sequence (5.2) and the boundary operator $\partial$, note that $\widetilde{C F}_{*}(Y) \cong C F_{*}\left(Y^{\prime}\right) \oplus C F_{*}\left(Y^{\prime \prime}\right)$. According to the previous discussion, we know that there is a homomorphism $R$ which is the right inverse of the homomorphism $\tilde{b}$. We may define a homomorphism $\triangle$ from $C F_{*}\left(Y^{\prime \prime}\right)$ to $C F_{*-1}\left(Y^{\prime}\right)$ with grading shift -1 as follows:

$$
\triangle=\tilde{a}_{1}^{-1} \partial R .
$$

It is easy to see that $\Delta$ is well defined and $\Delta_{*}$ is actually induced by the boundary operator $\partial$. Note that for any $\rho \in H F_{*}\left(Y^{\prime \prime}\right), \partial R(\rho) \in \overline{C F}_{*-1}(Y)$, in particular $\partial R(\rho)$ belongs to the image of the homomorphism $a_{1}$ since $\tilde{b} \partial R(\rho)=$ $\partial \widetilde{b} R(\rho)=\partial(\rho)=0$, and the kernel of $\tilde{b}$ is equal to the image of $\tilde{a}_{1}$. Also $\tilde{a}_{1}$ is an injective map which is chain homotopic to $i+\alpha$, where $i$ is the inclusion from $C F_{*}\left(Y^{\prime}\right)$ to $\widetilde{C F}_{*}(Y) \cong C F_{*}\left(Y^{\prime}\right) \oplus C F_{*}\left(Y^{\prime \prime}\right)$, thus there exists a unique element $\sigma \in C F_{*-1}\left(Y^{\prime}\right)$ such that $\tilde{a}_{1}(\sigma)=\partial R(\rho)$. Hence $\partial \widetilde{a}_{1}(\sigma)=\partial^{2} R(\rho)=0$, which implies that $\widetilde{a}_{1}(\partial \sigma)=\partial \widetilde{a}_{1}(\sigma)=0$ since $\tilde{a}_{1}$ is chain homotopic to the homomorphism $\tilde{a}$ which is induced by the cobordism $W_{1}$ and the deformed instanton equation on $W_{1}$, and $\partial \sigma=0$, that is, $\partial \widetilde{a}_{1}^{-1} \partial R(\rho)=0$. So $\sigma=\widetilde{a}_{1}^{-1} \partial R(\rho)$ $\in H F_{*-1}\left(Y^{\prime}\right)$ and $\triangle_{*}$ is the homomorphism from $H F_{*}\left(Y^{\prime \prime}\right)$ to $H F_{*-1}\left(Y^{\prime}\right)$ induced by the boundary operator $\partial$ and the short exact sequence (5.2).

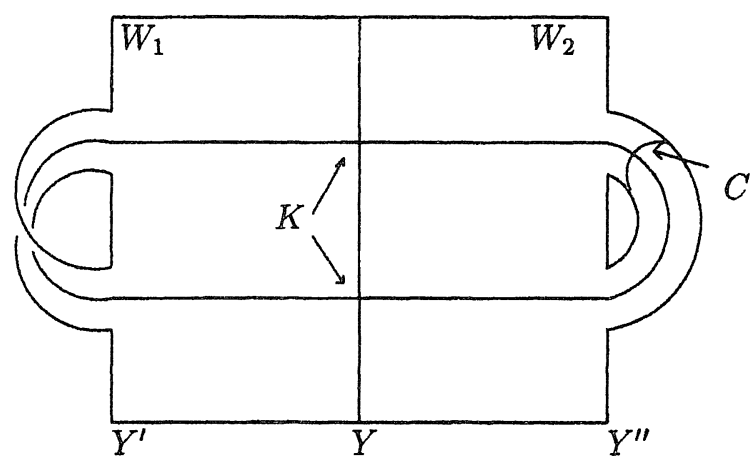

Figure 3 
Next we investigate the right inverse, $R$, of the homomorphism $\widetilde{b}$ induced by the cobordism $W_{2}$. It is clear that $R$ is a homomorphism from $C F_{*}\left(Y^{\prime \prime}\right)$ to $\widetilde{C F}_{*}$ $(Y)$ which is not induced by a zero dimensional $A S D$ moduli space over a cobordism. To find the corresponding cobordism, let us explain the construction of $R$. We know that there are the standard, oriented, surgery cobordisms $W_{1}$ from $Y^{\prime}$ to $Y$ and $W_{2}$ from $Y$ to $Y^{\prime \prime}$ which are illustrated in Figure 3. In this picture the dimensions have been reduced by two, so we see 2-manifolds with 1-dimensional boundaries, but it provides a fairly accurate guide to the higher dimensional case. In particular, if we let $\widehat{W}_{2}$ be the manifold obtained from $W_{2}$ by removing an interior point (Note that we let $C$ and $D_{2}$ intersect at the puncture point.). Let $Q_{2}$ be the ball with a metric having one tubular end, so that we can regard $W_{2}$ as the connected sum of $\widehat{W}_{2}$ and $Q_{2}$ across the $S^{3}$ ends (see Figure 4 ). If we choose any deformation of the instanton equation over $Q_{2}$ compatible with $\mathscr{C} \&+\Phi$ on the end, we can glue this to the time-independent deformation over $\widehat{W}_{2}$ to get a deformed instanton equation over $W_{2}$, defining a homomorphism $R: C F *\left(Y^{\prime \prime}\right) \rightarrow \overline{C F}_{*}(Y)$.

The complement $W_{2} \backslash D_{2}$ retracts onto the boundary component $Y^{\prime \prime}$, so any flat connection $\rho^{\prime \prime}$ over $Y^{\prime \prime}$ automatically extends to $W_{2} \backslash D_{2}$, that is, to the complement of the product region in $\widehat{W}_{2}$. Further more, by the construction of $\Phi$ (for more details, see [6]), this connection extends over the product region to a deformed-flat solution, say $A_{\rho^{\prime \prime}}$, of the deformed equations. Let $\widetilde{\rho}^{\prime \prime}$ be the restriction of $A_{\rho^{\prime \prime}}$ to $Y$. Using modified monotonicity of Chern-Simons, we have that $\mathscr{C} \&\left(\rho^{\prime \prime}\right)=(\mathscr{C} \&+\Phi)\left(\widetilde{\rho}^{\prime \prime}\right)$ modulo integers. Let $\nu\left(\rho^{\prime \prime}\right)$ be the parameter defining the restriction of $A_{\rho^{\prime \prime}}$ to the $S^{3}$ boundary, let $\sigma_{\nu}$ be the corresponding solution of the deformed equation on $S^{3}$ which is just an extension of a reducible flat connection over $S^{3} \backslash S^{1}$. Then we appeal to the gluing theory extended to the deformed equations. We can now use the following construction: For each flat connection $\rho^{\prime \prime}$ over $Y^{\prime \prime}$ let $A^{*}\left(\rho^{\prime \prime}\right)=$ $A_{\rho^{\prime \prime}} \# B\left(\nu\left(\rho^{\prime \prime}\right)\right)$ be the connection formed by gluing together the deformed-flat solution $A \rho^{\prime \prime}$ over $\widehat{W}_{2}$ and a reducible solution $B\left(\nu\left(\rho^{\prime \prime}\right)\right)$ to the deformed equation on $Q_{2}$, asymptotic to $\sigma_{\nu}$ over the end of $S^{3}$ of $Q_{2}$. It is similar to the result in [6] that we get a solution with the following properties:

(1) $A^{*}\left(\rho^{\prime \prime}\right)$ is irreducible (Since $A_{\rho^{\prime \prime}}$ is.).

(2) ind $A^{*}\left(\rho^{\prime \prime}\right)=1$.

(3) $A^{*}\left(\rho^{\prime \prime}\right)$ is a point in the corresponding smooth moduli space.

It follows first, from the index calculation (cf. Section 2), that ind $A^{*}\left(\rho^{\prime \prime}\right)$ must be one since one requires that the Floer-grading of the flat connection $\rho^{\prime \prime}$ over $Y^{\prime \prime}$ and of the deformed solution $\widetilde{\rho}^{\prime \prime}$ over $Y$ are the same, also the ordering of the flat connections $\rho^{\prime \prime}$ over $Y^{\prime \prime}$ induced by the Chern-Simons functional and the ordering of the deformed solutions $\widetilde{\rho}^{\prime \prime}$ by the deformed functional over $Y$ are 
the same. It is easy to see that the moduli space $M\left(\rho^{\prime \prime}, \widetilde{\rho}^{\prime \prime}\right)$ of the index one solutions over $\bar{W}_{2}$ interpolating between $\rho^{\prime \prime}$ and $\widetilde{\rho}^{\prime \prime}$ is smooth where $\bar{W}_{2}$ is the cobordism $W_{2}$ with the reversed orientation, i.e., an oriented cobordism from $Y^{\prime \prime}$ to $Y$. By the construction of the moduli space $\mathcal{M}_{\bar{W}}\left(\rho^{\prime \prime}, \widetilde{\rho}^{\prime \prime}\right)$, it can be regarded as the inverse procedure of the construction of the homomorphism $\widetilde{b}$ induced by the cobordism $W_{2}$, thus the base manifold of the moduli space $\mathcal{M}\left(\rho^{\prime \prime}, \widetilde{\rho}^{\prime \prime}\right)$ must be the cobordism $\bar{W}_{2}$ with the reversed orientation of $W_{2}$. Hence the right inverse $R$ of $\tilde{b}$ is chain homotopic to the following map:

$$
C F_{*}\left(Y^{\prime \prime}\right) \ni \rho^{\prime \prime} \mapsto \sum_{\substack{\operatorname{dim} \cdot \mathcal{M}\left(\rho^{\prime \prime} . \tilde{\rho}^{\prime \prime}\right)=1 \\ \tilde{\rho}^{\prime \prime} \in \widetilde{C} F_{+}(1)}}\left[\widetilde{\rho}^{\prime \prime}\right]
$$

where the summation is taken over one $\widetilde{\rho}^{\prime \prime}$ from each component of the moduli space $\mathcal{M}\left(\rho^{\prime \prime}, \widetilde{\rho}^{\prime \prime}\right)$ over $\bar{W}_{2}$ with dimension one. Note that in general the moduli space $\mathcal{M}\left(\rho^{\prime \prime}, \widetilde{\rho}^{\prime \prime}\right)$ is an one dimensional smooth manifold which may have different components. But the corresponding complex map $R$ is chain romotopic to the inclusion from $C F_{*}\left(Y^{\prime \prime}\right)$ into $\overline{C F}_{*}(Y) \cong C F_{*}\left(Y^{\prime}\right) \oplus C F_{*}\left(Y^{\prime \prime}\right)$, that is, the difference between $R$ and the inclusion from $C F_{*}\left(Y^{\prime \prime}\right)$ into $\widetilde{C F}_{*}(Y)$ is a strictly decreasing complex map with respect to the filtration of the Floer complexes, the proof is similar to the proof for the properties of $\widetilde{a}$ in [6].

In summary of the above discussion, we have the following technical lemma whose proof is omitted:

Lemma 5.1. The right inverse $R$ of the homomorphism $\tilde{b}$ induced by the cobordism $W_{2}$ is chain homotopic to the complex map (5.5) between $C F_{*}\left(Y^{\prime \prime}\right)$ and $\widetilde{C F}_{*}(Y)$ which is induced by the one dimensional moduli space of the dcformed ASD connections oner $\bar{W}_{2}$.

We would like to point out here that similarly, the preimage $\tilde{a}_{1}^{-1}$, of the homomorphism $\widetilde{a}_{1}$ is chain homotopic to the following map which is induced by the one dimensional moduli space of the deformed $A S D$ connections over $\bar{W}_{1}$, where $\bar{W}_{1}$ is an oriented cobordism from $Y$ to $Y^{\prime}$ with the reversed orientation of $W_{1}$ :

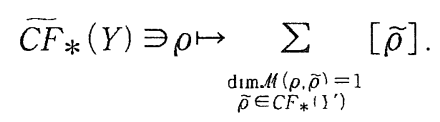

Notice that the summation is taken over one $\widetilde{\rho}$ from each component of the deformed moduli space $\mathcal{M}(\rho, \widetilde{\rho})$ over $\bar{W}_{1}$ with the dimension one (cf. Figure 4 ). 
Also the boundary operator $\partial$ on the complex $\overline{C F}_{*}(Y)$ is induced by an one dimensional deformed $A S D$ moduli space over the tube $Y \times R$. According to the above discussion, we have the following proposition:

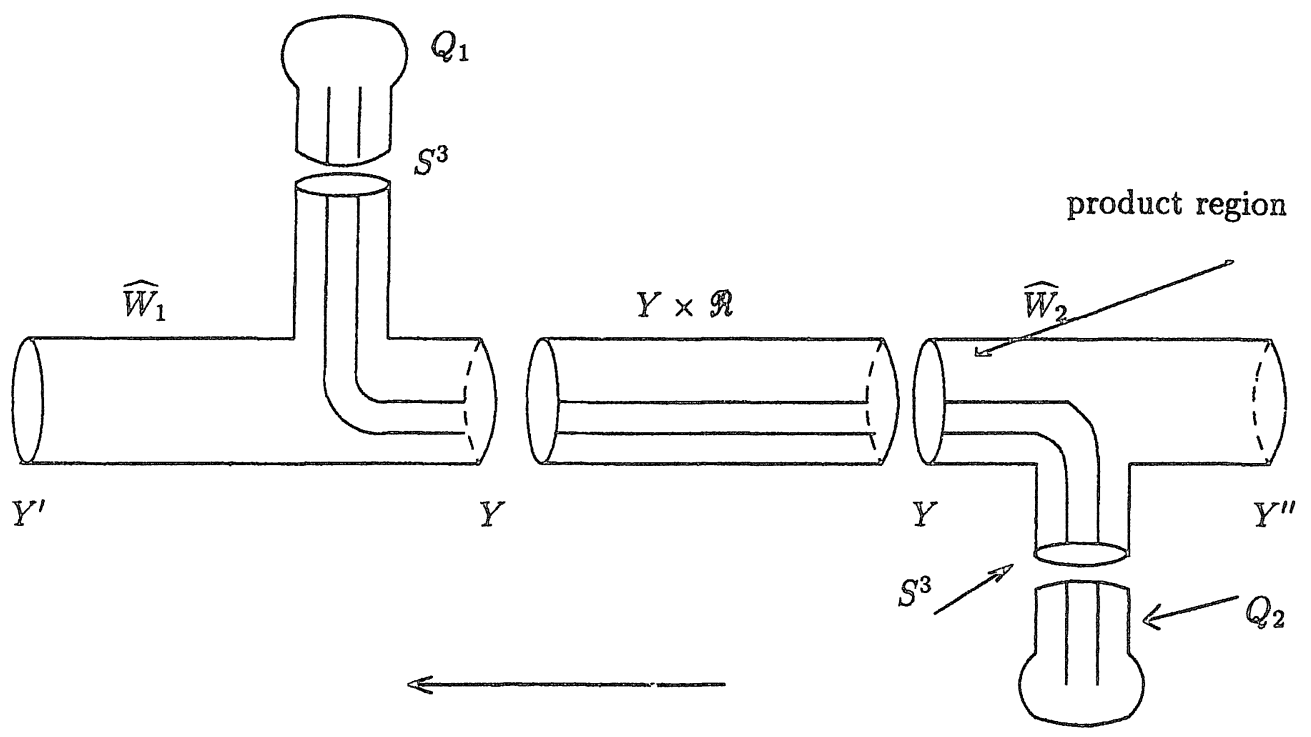

Figure 4

Proposition 5.2. The homomorphism $\triangle=\tilde{a}_{1}^{-1} \circ \partial \circ R$ is chain homotopic to the following map from $C F_{*}\left(Y^{\prime \prime}\right)$ to $C F_{*-1}\left(Y^{\prime}\right)$ :

$$
C F *\left(Y^{\prime \prime}\right) \ni \rho^{\prime \prime} \mapsto \sum_{\substack{\left.\operatorname{dim} \mathcal{M}\left(\rho^{\prime \prime}, \rho^{\prime}\right)=3 \\ \rho^{\prime} \in C F_{*-1} \in Y^{\prime}\right)}}\left[\tilde{\rho}^{\prime}\right] .
$$

Where the summation is taken over one $\rho^{\prime} \in C F_{*-1}\left(Y^{\prime}\right)$ for each component of the deformed $A S D$ moduli space $M\left(\rho^{\prime \prime}, \rho^{\prime}\right)$ over $\bar{W}_{2} \bar{W}_{1}$ with the dimension 3.

Remark that the homomorphism $\triangle=\tilde{a}_{1}^{-1} \circ \partial \circ R$ is just given by the 3dimensional ASD moduli space over $\bar{W}_{2} \bar{W}_{1}$. This is based on the following reason: The gluing of, for instance, 2, 1 and 0 -dimensional ASD moduli space over $\bar{W}_{2}, Y \times \mathscr{R}$ and $\bar{W}_{1}$ respectively would also give a 3 -dimensional ASD moduli over $\bar{W}_{2} \bar{W}_{1}$, but it is impossible. Suppose that a component of 3dimensional ASD moduli space, $M_{\vec{W}_{2} \bar{W}_{1}}\left(\rho^{\prime \prime}, \rho^{\prime}\right)$, is obtained from ASD moduli spaces $\mathcal{M}\left(\rho^{\prime \prime}, A_{\bar{W}_{2}}, \widetilde{\rho}^{\prime \prime}\right), \mathcal{M}\left(\widetilde{\rho}^{\prime \prime}, A_{Y \times \mathscr{R}}, \widetilde{\rho}^{\prime}\right)$ and $\mathcal{M}\left(\widetilde{\rho}^{\prime}, A_{\bar{W}_{1}}, \rho^{\prime}\right)$ over $\bar{W}_{2}, Y \times \mathscr{R}$ and $\bar{W}_{1}$ respectively. Since $b_{1}\left(Y^{\prime \prime}\right)=1, b_{2}^{+}\left(\bar{W}_{2}\right)=0, b_{2}^{+}\left(\bar{W}_{1}\right)=1$ and $Y, Y^{\prime}$ are homology 3 -spheres. Then by using index calculation in Section 2 , we have that 


$$
\begin{aligned}
\operatorname{dim} M\left(\rho^{\prime \prime}, A_{\bar{W}_{2}}, \widetilde{\rho}^{\prime \prime}\right) & =2 k\left(\rho^{\prime \prime}, A_{\bar{W}_{2}}, \widetilde{\rho}^{\prime \prime}\right)-3+\mu\left(\rho^{\prime \prime}\right)-\mu\left(\widetilde{\rho}^{\prime \prime}\right), \\
\operatorname{dim} \mathcal{M}\left(\widetilde{\rho}^{\prime \prime}, A_{Y \times \mathscr{R}}, \widetilde{\rho}^{\prime}\right) & =\mu\left(\tilde{\rho}^{\prime \prime}\right)-\mu\left(\widetilde{\rho}^{\prime}\right), \\
\operatorname{dim} M\left(\widetilde{\rho}^{\prime}, A_{\bar{W}_{1}}, \rho^{\prime}\right) & =2 k\left(\widetilde{\rho}^{\prime}, A_{\bar{W}_{1}}, \rho^{\prime}\right)-3+\mu\left(\widetilde{\rho}^{\prime}\right)-\mu\left(\rho^{\prime}\right) .
\end{aligned}
$$

On the other hand, $A_{\bar{W}_{2}}, A_{Y \times \Re}$ and $A_{\bar{W}_{1}}$ are irreducible ASD connections. From the monotonicity of the cobordism functor (see [6]), it follows that $k\left(\rho^{\prime \prime}, A_{\bar{W}_{2}}, \widetilde{\rho}^{\prime \prime}\right)$ and $k\left(\widetilde{\rho}^{\prime}, A_{\bar{W}_{1}}, \rho^{\prime}\right)$ must be 2 . Also, we have that

$$
\operatorname{dim} \mathcal{M}\left(\rho^{\prime \prime}, A_{\bar{W}_{2}}, \widetilde{\rho}^{\prime \prime}\right) \geq 1, \quad \operatorname{dim} \mathcal{M}\left(\widetilde{\rho}^{\prime}, A_{\bar{W}_{1}}, \rho^{\prime}\right) \geq 1 .
$$

Therefore, when $\operatorname{dim} \mathcal{M}\left(\widetilde{\rho}^{\prime \prime}, A_{Y^{\prime} \times \Re} . \widetilde{\rho}^{\prime}\right)=1$, then

$$
\operatorname{dim} \mathcal{M}\left(\rho^{\prime \prime}, A_{\bar{W}_{2}}, \widetilde{\rho}^{\prime \prime}\right)=1, \quad \operatorname{dim} \mathcal{M}\left(\widetilde{\rho}^{\prime}, A_{\bar{W}_{1}}, \rho^{\prime}\right)=1 .
$$

When $\operatorname{dim} M\left(\widetilde{\rho}^{\prime \prime}, A_{Y \times \Re}, \widetilde{\rho}^{\prime}\right)=0$, it follows that $\widetilde{\rho}^{\prime \prime} \cong \widetilde{\rho}^{\prime}$ and $A_{Y \times \Re}$ is induced by $\widetilde{\rho}^{\prime \prime}$. For this case, we have two possibilities: (1) $\operatorname{dim} \mathcal{M}\left(\rho^{\prime \prime}, A_{\bar{W}_{2}}, \widetilde{\rho}^{\prime \prime}\right)=2$, $\operatorname{dim}$ $\mathcal{M}\left(\tilde{\rho}^{\prime}, A_{\bar{W}_{1}}, \rho^{\prime}\right)=1$. Then $\mathcal{M}\left(\rho^{\prime \prime}, A_{\bar{W}_{2}}, \widetilde{\rho}^{\prime \prime}\right)$ defines the homomorphism $\partial \circ R$ and $\mathcal{M}\left(\widetilde{\rho}^{\prime}, A_{\bar{W}_{1}}, \rho^{\prime}\right)$ defines the homomorphism $\tilde{a}_{1}^{-1}$. (2) Similarly, if $\operatorname{dim} \mathcal{M}\left(\rho^{\prime \prime}\right.$, $\left.A_{\bar{W}_{2}}, \tilde{\rho}^{\prime \prime}\right)=1$ and $\operatorname{dim} \mathcal{M}\left(\widetilde{\rho}^{\prime}, A_{\bar{W}_{2}}, \rho^{\prime}\right)=2$. Then $\mathcal{M}\left(\rho^{\prime \prime}, A_{\bar{W}_{2}}, \widetilde{\rho}^{\prime \prime}\right)$ defines the homomorphism $R$ and $\mathcal{M}\left(\widetilde{\rho}^{\prime}, A_{\bar{w}_{1}}, \rho^{\prime}\right)$ defines the homomorphism $\tilde{a}_{1}{ }^{\circ} \partial$. Hence, the 3 -dimensional ASD moduli space, $\mathcal{M}_{\bar{W}_{2} \bar{W}_{1}}\left(\rho^{\prime \prime}, \rho^{\prime}\right)$, over $\bar{W}_{2} \bar{W}_{1}$ induces the homomorphism $\Delta=\tilde{a}_{1}^{-1} \circ \partial \circ R$. By the above discussion, we can count the number of the components of the 3 -dimensional ASD moduli space over $\bar{W}_{2} \bar{W}_{1}$ with sign defined by natural orientation. Actually, we will see that there exists a correspondence between each component of the 3-dimensional ASD moduli space, $M_{\bar{W}_{2} \bar{W}_{1}}\left(\rho^{\prime \prime}, \rho^{\prime}\right)$, over $\bar{W}_{2} \bar{W}_{1}$ and an isolated point of the 0 -dimensional ASD moduli space, $\mathcal{M}_{W_{3}}\left(\rho^{\prime \prime}, \rho^{\prime}\right)$, over $W_{3}$. Hence, we may count the number of the components of the 3-dimensional ASD moduli space over $\bar{W}_{2} \bar{W}_{1}$ with sign by counting the number of the points of the 0 -dimensional ASD moduli space over $W_{3}$.

With Proposition 5.2 understood, we can now go to the proof of the main theorem. Here we use a similar idea to the vanishing argument in [6], [11].

Proof of the main theorem. According to Proposition 5.2, the homomorphism $\triangle=\tilde{a}_{1}^{-1} \partial R$ is chain homotopic to the map which is induced from a 3-dimensional deformed $A S D$ moduli space $\mathcal{M}\left(\rho^{\prime \prime}, \rho^{\prime}\right)$ over the composite cobordism $\bar{W}_{2} \bar{W}_{1}$ from $Y^{\prime \prime}$ to $Y^{\prime}$, where $\rho^{\prime \prime} \in C F_{*}\left(Y^{\prime \prime}\right) \rho^{\prime} \in C F *-1\left(Y^{\prime}\right)$. Recall the constructions of $W_{1}$ and $W_{2}$, it is easy to see that there is an embedded 2-sphere $\bar{\Sigma}$ in the composite cobordism $\bar{W}_{2} \bar{W}_{1}$ from $Y^{\prime \prime}$ to $Y^{\prime}$, with selfintersection +1 . The boundary of a tubular neighborhood of $\bar{\Sigma}$ is a 3 -sphere, and we can write

$$
\bar{W}_{2} \bar{W}_{1}=W_{3} \# \boldsymbol{C} P^{2} \text {, }
$$


where $W_{3}$ is another cobordism from $Y^{\prime \prime}$ to $Y^{\prime}$ which induces the homomorphism $c *$.

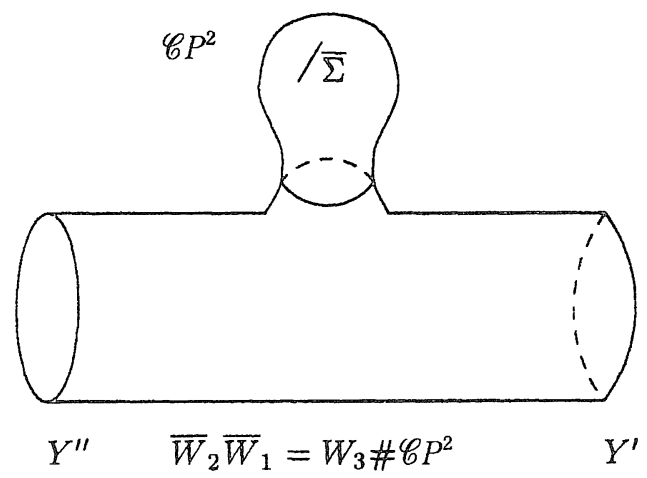

Figure 5

Note that the homomorphism $\Delta=\tilde{a}_{1}^{-1} \circ \partial \circ R$ is induced by counting the number of the components of the 3 -dimensional $A S D$ moduli space over the composite cobordism $\bar{W}_{2} \bar{W}_{1}$ with sign. Since $\Delta=\tilde{a}_{1}^{-1} \circ \partial \circ R$ is the composite homomorphism. Thus we may assume that $\Delta$ is independent of the metric on $\bar{W}_{2} \bar{W}_{1}$. We consider a family of metrics $g_{\lambda}, \lambda \rightarrow 0$ on $\bar{W}_{2} \bar{W}_{1}$, that is, the neck of the connected sum (cf. Figure $5, \bar{W}_{2} \bar{W}_{1}=W_{3} \# \mathbb{C} P^{2}$ ) is made very small, or what is conformally the same, the manifolds are joined by a very long tube. Then we claim that for small $\lambda$ the relevant 3 -dimensional moduli space of $A S D$ connections comes from the moduli spaces over $W_{3}$ and $\mathscr{C} P^{2}$. Informally, such instantons over $\bar{W}_{2} \bar{W}_{1}$ are made up by gluing an $S O(3)$-instanton $A_{W_{3}}$ over $W_{3}$ to an $S O(3)$-instanton, with non-zero second Stiefel-Whitney class, $A_{C P^{2}}$ over $\mathscr{C} P^{2}$. The instanton $A_{W_{3}}$ is irreducible, since it is already irreducible on the boundary, hence its deformation index, say ind $\left(A_{W_{3}}\right)$, is non-negative. The instanton over $\mathbb{C} P^{2}$ must be irreducible since the second Stiefel-Whitney class is non-zero. The addition rule for the index tells us that a connection $A_{\bar{W}_{2} \mid \bar{W}_{1}}=$ $A_{W_{3}} \# A_{\boldsymbol{C} P^{\prime}}$ made by gluing these together has the index

$$
\text { ind }\left(A_{\bar{w}_{2}, \bar{V}_{2}}\right)=\text { ind }\left(A_{W_{3}}\right)+\operatorname{ind}\left(A_{C P^{2}}\right)+3 \geq 3
$$

so in particular such instantons induced the homomorphism $\Delta$ are constructed by the instantons with index zero over $W_{3}$ and $\mathscr{C} P^{2}$. More precisely, the indices are given as follows:

$$
\text { ind } \begin{aligned}
\left(A_{\bar{W}_{2} \bar{W}_{1}}\right) & =2 k\left(\rho^{\prime \prime}, A_{\bar{W}_{2} \bar{W}_{1}}, \rho^{\prime}\right)-3 \chi^{+}\left(\bar{W}_{2} \bar{W}_{1}\right)+\mu\left(\rho^{\prime \prime}\right)-\mu\left(\rho^{\prime}\right) \\
& =2 k\left(\rho^{\prime \prime}, A_{W_{2}, \bar{W}_{1}}, \rho^{\prime}\right)-6+1
\end{aligned}
$$




$$
\begin{aligned}
& =2 k\left(\rho^{\prime \prime}, A_{\bar{W}_{2} \bar{W}_{1}}, \rho^{\prime}\right)-5, \\
\operatorname{ind}\left(A_{C P^{2}}\right) & =2 k\left(A_{C P^{2}}\right)-6, \\
\text { ind }\left(A_{W_{3}}\right) & =2 k\left(\rho^{\prime \prime}, A_{W_{3}}, \rho^{\prime}\right)-3 \chi^{+}\left(W_{3}\right)+\mu\left(\rho^{\prime \prime}\right)-\mu\left(\rho^{\prime}\right) \\
& =2 k\left(\rho^{\prime \prime}, A_{W_{3}}, \rho^{\prime}\right)-2 .
\end{aligned}
$$

Hence, we may take $k\left(\rho^{\prime \prime}, A_{\bar{W}_{2} \bar{W}_{1}}, \rho^{\prime}\right)=4, k\left(A_{C P^{2}}\right)=3$ and $k\left(\rho^{\prime \prime}, A_{W_{3}}, \rho^{\prime}\right)=1$ such that ind $\left(A_{\bar{W}_{2} \bar{W}_{1}}\right)=3$, ind $\left(A_{C P^{2}}\right)=0$, ind $\left(A_{W_{3}}\right)=0$.

In the above inequality (5.8), the number 3 means the dimension of the gluing parameters, in fact, the gluing parameters are $S O(3)$. On the other hand, it is well known that the zero dimensional $A S D$ moduli space with the structure group $S O(3)$ over $C P^{2}$ is a single point (see 4.1 .4 in [11]). Hence it is clear that there exists a correspondence between the three dimensional $A S D$ moduli space over the composite cobordism $\bar{W}_{2} \bar{W}_{1}$ and the zero dimensional $A S D$ moduli space over the cobordism $W_{3}$. Therefore the homomorphism $\triangle=$ $\tilde{a}_{1}^{-1} \partial R$ induced by the three dimensional $A S D$ moduli space over $\bar{W}_{2} \bar{W}_{1}$ is chain homotopic to the homomorphism $c$ induced by the zero dimensional $A S D$ moduli space over $W_{3}$. Thus $\Delta_{*} c_{*}$. The proof of the main theorem is complete.

\section{A. Index Formulas}

In this appendix, give a proof of Proposition 2.6. Assume that $\rho$ and $\sigma$ are regular and nondegenerate flat connections over the 3 -manifolds $Y_{1}$ and $Y_{2}$ respectively. $A$ is an irreducible $A S D$ connection over a 4 -manifold $X$ which is of the radial gauge on the cylindrical ends with $A(t)$ converging to $\rho$ and $\sigma$ as $t$ tending to $-\infty$ and $+\infty$ respectively. The key point is that the operator $D_{A}^{+}$is closely related to the twisted signature operator

$$
d_{A}+d_{A}^{*}: \Omega^{0} \oplus \Omega^{1} \oplus \Omega_{+}^{2} \longrightarrow \Omega^{0} \oplus \Omega^{1} \oplus \Omega_{-}^{2} .
$$

One casily checks that the signature operator is given by

$$
(\xi, \eta, \zeta) \longmapsto\left(d_{A}^{*} \eta, d_{A} \xi+d_{A}^{*} \zeta, d_{A}^{-} \eta\right) .
$$

Thus in terms of the operators

$$
D_{A}^{ \pm}=d_{A}^{*} \oplus d_{A}^{ \pm}: \Omega^{1} \longrightarrow \Omega^{0} \oplus \Omega_{ \pm}^{2},
$$

the twisted signature operator can be expressed as

$$
\left(\begin{array}{cc}
D_{A}^{-} & 0 \\
0 & \left(D_{A}^{+}\right)^{*}
\end{array}\right): \Omega^{0} \oplus \Omega^{1} \oplus \Omega_{+}^{2} \longrightarrow \Omega^{0} \oplus \Omega^{1} \oplus \Omega_{-}^{2} .
$$

Note that it follows from [4] that

$$
\operatorname{ind} D_{A}^{-}-\operatorname{ind} D_{A}^{+}=\int_{X}\left(4+\frac{p_{1}(X)}{3}\right) C h(A)+\eta(\rho)-\eta(\sigma)
$$


and

$$
\text { ind } D_{A}^{-}+\operatorname{ind} D_{A}^{+}=-\int_{X} e(X) C h(A)
$$

We find

$$
\operatorname{ind} D_{A}^{+}=-\frac{1}{2} \int_{X}\left(4+\frac{p_{1}(X)}{3}+e(X)\right) C h(A)+\frac{1}{2} \eta(\sigma)-\frac{1}{2} \eta(\rho),
$$

where

$$
C h(A)=\operatorname{dim} S O(3)+\frac{-1}{8 \pi^{2}} \operatorname{tr}\left(F_{A} \wedge F_{A}\right)
$$

In terms of (A.6) and (A.7), we have

$$
\operatorname{ind} D_{A}^{+}=\frac{1}{4 \pi^{2}} \int_{X} \operatorname{tr}\left(F_{A} \wedge F_{A}\right)-\frac{3}{2} \int_{X}\left(\frac{p_{1}(X)}{3}+e(X)\right)+\frac{1}{2} \eta(\sigma)-\frac{1}{2} \eta(\rho) \text {. }
$$

Now we can prove Proposition 2.6 by using the formulae discussed above.

Proof of Proposition 2.6. Under the assumption, the cylindrical ends $\rho$ and $\sigma$ need not be acyclic. Thus 0 may appear in the spectrum of the operators $D_{A}=\frac{d}{d t}+D_{\rho}$ and $D_{A}=\frac{d}{d t}+D_{\sigma}$ over the tubular parts of $X$, and there are kernels of the positive dimension. To recover a Fredholm theory we use the weighted function spaces (cf. [26]. [28] and [30]).

Now we digress to discuss the operator $D_{A}$ over the weighted Sobolev space. We can now set up the index theory over a general 4-manifold $X$ with tubular ends $Y_{\imath} \times(0, \infty)$. We have to choose a weight $\alpha_{\imath}$ for each end $Y_{\imath} \times$ $(0, \infty)$. To be more precise we define the weighted $L^{2}$ norm on the sections of $\Gamma\left(A d P \otimes T^{*} X\right)$ over $X$ by

$$
\|f\|_{L^{2 \alpha}}^{2}=\int_{X}|w f|^{2}
$$

where $w$ is a non-vanishing function on $X$ which is equal to $e^{\alpha_{t t}}$ on the tubular ends $Y_{\imath} \times(0, \infty)$. That is: $\|f\|_{L^{2 \alpha}}=\|w f\|_{L^{2}}$. Similarly we define the weighted $L_{1}^{2}$ norm by

$$
\|f\|_{L_{1}^{2} \alpha}=\|w f\|_{L_{1}^{2}}
$$

Different choices of $w$ with the same weight vector $\alpha=\left(\alpha_{1}, \alpha_{2}, \cdots\right)$ give equivalent norms. Hence we can consider the operators on the weighted Sobolev spaces

$$
D_{A}^{ \pm} \cdot \alpha=d_{A, \alpha}^{*}+d_{A}^{ \pm}: L_{1}^{2, \alpha}\left(A d P \otimes T^{*} X\right) \longrightarrow L^{2, \alpha}(A d P) \oplus L^{2, \alpha}\left(A d P \otimes \wedge_{ \pm}^{2} T^{*} X\right) .
$$

Here $d_{A, \alpha}^{*}$ is the $L^{2, \alpha}$-adjoint of $d_{A}$. The operators $D_{A, \alpha}^{ \pm}$are Fredholm as long as 
$\alpha_{i}$ does not lie in the spectrum of the operator $D_{\rho_{i}}$ over $Y_{\imath}$. Also one can show that the index of the operator does not change if $\alpha$ is varied in such way that $\alpha_{i}$ avoids the spectrum of $D_{\rho_{s}}$ over $Y_{i}$. Conversely, the index will change if $\alpha_{i}$ is moved across an eigenvalue (cf. [26], [28] and [30]).

We return to the proof of Proposition 2.6. Under the assumption, $\rho=\left(\rho_{1}\right.$, $\left.\cdots, \rho_{m}\right)$ and $\sigma=\left(\sigma_{1}, \cdots, \sigma_{n}\right)$ are nondegenerate flat connection vectors over the 3-manifold vectors $Y_{1}=\left(Y_{11}, \cdots, Y_{1 m}\right), Y_{2}=\left(Y_{21}, \cdots, Y_{2 n}\right)$ respectively. Thus one may choose the two weight vectors $\alpha^{1}=\left(\alpha_{1}^{1}, \cdots, \alpha_{m}^{1}\right)$ and $\alpha^{2}=\left(\alpha_{1}^{2}, \cdots, \alpha_{n}^{2}\right)$ such that $0<\alpha_{i}^{1}<\delta_{i}^{1}$, for $1 \leq i \leq m$ and $0<\alpha_{j}^{2}<\delta_{j}^{2}$, for $1 \leq j \leq n$ where $\delta_{i}^{1}, 1 \leq i \leq m$ and $\delta_{j}^{2}, 1 \leq j \leq n$ are the minimum absolute values of the non-zero eigenvalues of $D_{\rho_{i}}, 1 \leq_{i} \leq_{m}$ and $D_{\sigma_{1}}, 1 \leq_{j} \leq_{n}$ respectively. Also one chooses a positive function $w$ which is equal to $e^{-\alpha_{t}^{2} t}$ over the tubular ends $Y_{1 \imath} \times(-\infty, 0)$ and equal to $e^{\alpha_{3}^{2} t}$ over the tubular end $Y_{2 j} \times(0, \infty)$ respectively. Then $D_{A, \alpha^{1}, \alpha^{2}}^{+}$is a Fredholm operator and there exists a generic metric $g$ on $X$ such that the moduli space $\bar{M}_{g, \alpha^{1}, \alpha^{2}}^{*}(\rho, P, \sigma)$ is a finite dimensional smooth manifold. Formally the tangent space to $\bar{M}_{g, \alpha^{1}, \alpha^{2}}^{*}(\rho, P, \sigma)$ at [A] consists of elements in the kernel of $D_{A, \alpha^{1}, \alpha^{2}}^{+}$which satisfy a suitablc decay condition. Hence the moduli space $\bar{M}_{g, \alpha^{1}, \alpha^{2}}^{*}(\rho, P, \sigma)$ consists of the $A S D$ connections on $P$ which, on the cylindrical ends, agree with $\rho$ and $\sigma$, modulo the gauge group $\mathscr{G}_{2,0}^{2}(P)$. The associated group $\mathscr{G}_{2,0}^{2}(P)$ consists of those sections $g$ of the adjoint bundle $A u t P$ such that

$$
g^{-1} d g \in L_{1}^{2, \alpha}(A d P), \lim _{t \rightarrow \pm \infty} g(t) \in \mathscr{Z}(G) .
$$

Recall that

$$
\operatorname{Sign}(X)=b_{2}^{+}-b_{2}^{-}=\int_{X} \frac{p_{1}}{3}+\eta\left(Y_{2}\right)-\eta\left(Y_{1}\right) .
$$

and the index of the operator $D_{A, \alpha^{1}, \alpha^{2}}[4]$ is given by

$$
\text { ind } \begin{aligned}
D_{A, \alpha^{1}, \alpha^{2}}^{+}= & \frac{1}{4 \pi^{2}} \int_{X} \operatorname{tr}\left(F_{A} \wedge F_{A}\right)-\frac{3}{2} \int_{X}\left(\frac{p_{1}}{3}+e\right)+\frac{1}{2} \eta(\sigma)-\frac{1}{2} \eta(\rho) \\
& +\frac{1}{2} \operatorname{dim} \mathscr{G}_{\sigma}+\frac{1}{2} \operatorname{dim} \mathscr{G}_{p} .
\end{aligned}
$$

Where $\eta\left(Y_{1}\right), \eta\left(Y_{2}\right)$ are eta invariants of the corresponding operators on the scalar forms over the 3-manifolds $Y_{1}$ and $Y_{2}$ respectively. Combining (A.9). (A.13) and (A.14) together, in terms of the definitions of $\tilde{\eta}, \mu, k(\sigma, A, \rho)$ in $\S 2$, one has 


$$
\begin{aligned}
\operatorname{dim} \bar{M}_{g, \alpha^{1}, \alpha^{2}}(\rho, P, \sigma)= & \frac{1}{4 \pi^{2}} \int_{X} \operatorname{tr}\left(F_{A} \wedge F_{A}\right) \\
& -\frac{3}{2}[\operatorname{Sign}(X)+\chi(X)]+\frac{1}{2} \widetilde{\eta}(\sigma)-\frac{1}{2} \widetilde{\eta}(\rho) \\
& \quad+\frac{1}{2} \operatorname{dim} \mathscr{G}_{\sigma}+\frac{1}{2} \operatorname{dim} \mathscr{G}_{\rho} \\
= & 2 k(\rho, A, \sigma)+\mu(\rho)-\mu(\sigma)-\frac{3}{2}[\operatorname{Sign}(X)+\chi(X)] \\
& +\operatorname{dim} \mathscr{G}_{\rho}+\frac{3}{2}\left[b_{0}\left(Y_{2}\right)+b_{1}\left(Y_{2}\right)-b_{0}\left(Y_{1}\right)-b_{1}\left(Y_{1}\right)\right] .
\end{aligned}
$$

Now the exact sequence of $(X, \partial X)$ with $\partial X=Y=Y_{1} \cup Y_{2}$ yields the identity

$$
\begin{aligned}
& b_{2}(X)-b_{2}^{+}(X)-b_{2}^{-}(X)= \\
& \quad b_{0}(X)-b_{1}(X)+b_{3}(X)-b_{4}(X)-b_{0}(Y)+b_{1}(Y),
\end{aligned}
$$

thus.

$$
\begin{aligned}
\operatorname{Sign}(X)+\chi(X)= & b_{2}^{+}(X)-b_{2}^{-}(X)+b_{0}(X)+b_{2}(X) \\
& +b_{4}(X)-b_{1}(X)-b_{3}(X) \\
= & b_{2}^{+}(X)-b_{2}^{-}(X)+b_{0}(X) \\
& +\left\{b_{0}(X)-b_{1}(X)+b_{2}^{+}(X)+b_{2}^{-}(X)\right. \\
& \left.\quad+b_{3}(X)-b_{4}(X)-b_{0}(Y)+b_{1}(Y)\right\} \\
& \quad+b_{4}(X)-b_{1}(X)-b_{3}(X) \\
= & 2 b_{2}^{+}(X)+2 b_{0}(X)-2 b_{1}(X)-b_{0}(Y)+b_{1}(Y) \\
= & 2 b_{2}^{+}(X)+2 b_{0}(X)-2 b_{1}(X) \\
& -b_{0}\left(Y_{1}\right)-b_{0}\left(Y_{2}\right)+b_{1}\left(Y_{1}\right)+b_{1}\left(Y_{2}\right) .
\end{aligned}
$$

Therefore, one has

$$
\begin{aligned}
\operatorname{dim} \bar{M}_{g, \alpha^{1}, \alpha^{2}}(\rho, P, \sigma)=2 k & (\rho, A, \sigma)+\mu(\rho)-\mu(\sigma)+\operatorname{dim} \mathscr{G}_{\rho} \\
& -3\left[b_{2}^{+}(X)+b_{0}(X)-b_{1}(X)-b_{0}\left(Y_{2}\right)+b_{1}\left(Y_{1}\right)\right] .
\end{aligned}
$$

Hence.

$$
\operatorname{dim} \bar{M}_{g, \alpha^{1}, \alpha^{2}}(\rho, P, \sigma)=2 k(\rho, A, \sigma)+\mu(\rho)-\mu(\sigma)+\operatorname{dim} g_{\rho}-3 \chi^{+}(X) . \text { (A.15) }
$$

According to the definitions of $\mathcal{M}_{g}^{*}(\rho, P, \sigma)$ and $\bar{M}_{g, \alpha^{1}, \alpha^{2}}(\rho, P, \sigma)$, one has

$$
\mathcal{M}_{g}^{*}(\rho, P, \sigma)=\bar{M}_{g . \alpha^{1}, \alpha^{2}}^{*}(\rho, P, \sigma) / \mathscr{G}_{\rho} \times \mathscr{G}_{\sigma} .
$$

Therefore, combining (A.15) and (A.16) together, we find the dimension of $\mathcal{M}_{g}^{*}$ $(\rho, P, \sigma)$ :

$$
\operatorname{dim} \mathcal{M}_{g}^{*}(\rho, P, \sigma)=2 k(\rho, P, \sigma)+\mu(\rho)-\mu(\sigma)-3 \chi^{+}(X)-\operatorname{dim} \mathscr{G}_{\sigma .}
$$


Thus, the proof of Proposition 2.6 is complete.

\section{References}

[1] Akbulut, S. and McCarthy, J. D., Casson's invariant for oriented homology 3-spheres, an exposition, Math. Notes 36. Princeton Univ. Press 1990.

[2] Atiyah, M. F., New invariants for 3 and 4-dimensional manifolds, Proc. Sympos. Pure Math., 48 (1988).

[3] Atiyah, M. F.. Bott, R. and Patodi, V. K., On the heat equation and the index theorem, Invent. Math., 19 (1973), 179-330.

[4] Atiyah. M. F., Patodi, V. K. and Singer. I. M., Spectral asymmetry and Riemannian geometry, 1. 2, 3, Math. Proc. Cambrndge Philos. Soc., 77 (1975a) 43-69, 78 (1975b) 405-432, 79 (1976) 71-99.

[5] Braam. P. J., Floer homology groups for homology 3-spheres, Adv. Math., 88 (1991), 131-144.

[6] Braam. P. J. and Donaldson, S. K.. Floer's work for instanton homology, knots and surgery. preprint.

[7] Chern, S. S. and Simons, J. Characterıstıc forms and geometric invariants. Ann. Math., 99 (1974), 48-69.

[8] Donaldson, S. K.. The orientation of Yang-Mills moduli spaces and 4-manifolds topology, $J$. Diff. Geom.. 26 (1987), 397-428.

[9] Polynomial invariants for smooth 4-manifolds, Topology, 29 (1990), 257-315.

[10] Donaldson, S. K., Furuta. M. and Kotschick. D., Floer homology groups in Yang-Mills theory, to appear

[11] Donaldson, S. K. and Kronheimer, P. B., The geometry of 4-manifolds. Oxford Unıv. Press 1990.

[12] Dostoglou, S. and Salamon. D.. Instanton homology and symplectic fixed points, preprint.

[13] - Self-dual instantons and holomorphic curves, preprint.

[14] Fintushel, R. and Stern, R J.. Using Floer's exact triangle to compute Donaldson invarıants. preprint.

[15] Floer, A., An instanton invariant for three-manifolds, Comm. Malh. Phys., 118 (1987). 215-240.

[16] Floer, A., Instanton homology, surgery and knots, in 'Geometry of Low-Dimensional Manıfolds 1'. pp 97-113, ed. by S. K. Donaldson and C. B. Thomas, LMS Lecture Note Seres 150. Cambrige Univ. Press 1990.

[17] Instanton homology and Dehn surgery, preprnnt.

[18] Instanton homology for knots. Prelimunary preprnt.

[19] Fukaya, K., Instanton homology for oriented 3-manifolds, in Adv. Stud. Pure Math, 20 'Aspccts of Lou' Dimensional Manfolds'. pp 1-92. ed. by Matsumoto, Morita 1992.

[20] Floer homology of connected sum of homology 3-spheres, preprint

[21] Kurby, R. A calculus for framed links in S3. Invent. Math., 45 (1978), 35-56.

[22] - The topology of 4-manıfolds, Lecture Notes in Math.. 1374. Sprunger Verlag 1989.

[23] Kotschick, D., SO (3) invarıants for 4-manifolds with $b_{2}^{+}=1$, Proc. Lond. Math. Soc., 63 (1991). 426-448.

[24] Kronheimer, P. B., Instanton invariants and flat connections on the Kummer surface, Duke Math. J, 64 (1991), 229-241.

[25] Kronheimer, P. B. and Mrowka, T. S.. Gauge theory for embedded surfaces I, II, preprint.

[26] Morgan. J. W., Mrowka. T. S. and Ruberman, D., The $L^{2}$-modulı space and a vanishing 
theorem for Donaldson polynomial invariants, preprint.

[27] Mrowka, T. S., A local Mayer-Vietoris principle for Yang-Mills moduli spaces, Harvard PhD thesis.

[28] Taubes, C. H., Gauge theory on asymptotically periodic 4-manifolds, J. Diff. Geom., 25 (1987), $363-430$

[29] - Casson's Invariant and gauge theory, J. Diff. Geom.., 31 (1990), 547-599.

[30] - $L^{2}$-moduli spaces on 4-manifolds with cylindrical ends, I. preprint.

[31] Uhlenbeck, K. K., Connections with $L^{2}$-bounds on curvature, Comm. Math. Phys., 83 (1982), 31-42.

[32] Witten, E., Supersymmetry and Morse theory. J. Diff. Geom., 17 (1982), 661-692. 